\title{
Lattice Representations with Set Partitions Induced by Pairings
}

\author{
Giampiero Chiaselotti \\ Department of Mathematics and Computer Science \\ University of Calabria \\ Arcavacata di Rende (CS), Italy \\ giampiero.chiaselotti@unical.it \\ Tommaso Gentile Federico Infusino \\ Department of Mathematics and Computer Science \\ University of Calabria \\ Arcavacata di Rende (CS), Italy \\ \{gentile,f.infusino\}@mat.unical.it
}

Submitted: Jun 10, 2019; Accepted: Jan 8, 2020; Published: Jan 24, 2020

(C) The authors. Released under the CC BY-ND license (International 4.0).

\begin{abstract}
We call a quadruple $\mathcal{W}:=\langle F, U, \Omega, \Lambda\rangle$, where $U$ and $\Omega$ are two given non-empty finite sets, $\Lambda$ is a non-empty set and $F$ is a map having domain $U \times \Omega$ and codomain $\Lambda$, a pairing on $\Omega$. With this structure we associate a set operator $M_{\mathcal{W}}$ by means of which it is possible to define a preorder $\geqslant_{\mathcal{W}}$ on the power set $\mathcal{P}(\Omega)$ preserving settheoretical union. The main results of our paper are two representation theorems. In the first theorem we show that for any finite lattice $\mathbb{L}$ there exist a finite set $\Omega_{\mathbb{L}}$ and a pairing $\mathcal{W}$ on $\Omega_{\mathbb{L}}$ such that the quotient of the preordered set $\left(\mathcal{P}\left(\Omega_{\mathbb{L}}\right), \geqslant \mathcal{W}\right)$ with respect to its symmetrization is a lattice that is order-isomorphic to $\mathbb{L}$. In the second result, we prove that when the lattice $\mathbb{L}$ is endowed with an order-reversing involutory map $\psi: L \rightarrow L$ such that $\psi\left(\hat{0}_{\mathbb{L}}\right)=\hat{1}_{\mathbb{L}}, \psi\left(\hat{1}_{\mathbb{L}}\right)=\hat{0}_{\mathbb{L}}, \psi(\alpha) \wedge \alpha=\hat{0}_{\mathbb{L}}$ and $\psi(\alpha) \vee \alpha=\hat{1}_{\mathbb{L}}$, there exist a finite set $\Omega_{\mathbb{L}, \psi}$ and a pairing on it inducing a specific poset which is order-isomorphic to $\mathbb{L}$.
\end{abstract}

Mathematics Subject Classifications: 06A07, 68R05

\section{Introduction}

Granular Computing (briefly $\mathrm{GrC}$ ) is an emerging paradigm which relies on the idea of partitioning a set of objects in some granules depending on some given criteria [29, $30,38,39]$. Many ideas and methods of $\mathrm{GrC}$ have been used in order to investigate 
discrete mathematical objects, such as matroids, set partitions and ordered structures $[21,25,26,36,37]$.

In particular, a natural partitioning of a finite object set $U$ is given when one uses a corresponding finite attribute set $\Omega$, with respect to which any ordered pair $(u, a) \in U \times \Omega$ takes a unique value $F(u, a)$ in a given set $\Lambda$. The quadruple $\langle F, U, \Omega, \Lambda\rangle$ has several names in computer science literature: information system [28], relational data table [34], information table [38], Chu space [20].

In this work we use some algebraic granular computing techniques on quadruples having the aforementioned form, in order to provide two representation results concerning finite lattices. However, due to the fact that in the present paper the above sets $U, \Omega$ and $\Lambda$ have only a formal nature, we will use the more mathematical term pairing instead of the previous ones. Hence, in our specific mathematical viewpoint, we can speak of algebraic GrC methods on pairings (for further results on such topics see also $[13,14,17]$ ).

Therefore, we shall call a quadruple $\mathcal{W}:=\langle F, U, \Omega, \Lambda\rangle$, where $U$ and $\Omega$ are both finite non-empty sets, $\Lambda$ is also a non-empty set and $F$ is a map having domain $U \times \Omega$ and codomain $\Lambda$, a pairing on $\Omega$. We may identify the pairing $\mathcal{W}$ with the rectangular table with rows labelled by the elements of $U$, the columns by those of $\Omega$ and whose entries are the values $F(u, a)$.

For any $A \subseteq \Omega$ we consider the following equivalence relation $\equiv_{A}$ on $U$ :

$$
u \equiv_{A} u^{\prime}: \Longleftrightarrow F(u, a)=F\left(u^{\prime}, a\right) \quad \forall a \in A .
$$

for any $u, u^{\prime} \in U$. Let $[u]_{A}$ be the equivalence class of $u$ with respect to $\equiv_{A}$ and $\pi_{\mathcal{W}}(A):=$ $\left\{[u]_{A}: u \in U\right\}$ the set partition on $U$ induced by $\equiv_{A}$. When we take as pairing $\mathcal{W}$ a data table having $\Omega$ as its attribute set, the equivalence relation $\equiv_{A}$ becomes a well-known tool of database theory and related fields $[28,34]$.

On the other hand, the relation $\equiv_{A}$ becomes a type of local symmetry relation with respect to a fixed vertex subset $A \subseteq V(G)$ when one interprets a finite simple undirected graph $G$ with vertex set $V(G)$ as a pairing $\langle F, V(G), V(G),\{0,1\}\rangle$ on $V(G)$, where $F(u, a):=1$ if the vertices $u, a \in V(G)$ are adjacent and $F(u, a):=0$ otherwise. In [13] such a local symmetry relation has been investigated for some basic graph families and, in particular, a classification theorem for the Petersen graph, concerning all subgraphs induced by the vertex subsets $A$ that are minimal with respect to the property that $\equiv_{A}$ agrees with $\equiv_{V(G)}$ has been proved (these vertex subsets have been called symmetry bases of the graph). Again, in [18] the local symmetry relation $\equiv_{A}$ has been studied in its interrelations with a specific type of binary operation o defined on the power set $\mathcal{P}(V(G))$ and whose automorphism group is isomorphic to a subgroup of $A u t(G)$ (for works on similar topics see also $[31,35])$.

Based on the particular graph interpretation introduced in [13], the same applies to the general case. Therefore, we call $\equiv_{A}$ the $A$-symmetry relation and $\pi_{\mathcal{W}}(A)$ the $A$-symmetry partition of the pairing $\mathcal{W}$. Moreover, we also consider the equivalence relation $\approx_{\mathcal{W}}$ on the power set $\mathcal{P}(\Omega)$ defined by

$$
A \approx_{\mathcal{W}} A^{\prime}: \Longleftrightarrow \pi_{\mathcal{W}}(A)=\pi_{\mathcal{W}}\left(A^{\prime}\right)
$$


for any $A, A^{\prime} \in \mathcal{P}(\Omega)$, that is equivalent to say that

$$
u \equiv_{A} u^{\prime} \Longleftrightarrow u \equiv_{A^{\prime}} u^{\prime}
$$

for all $u, u^{\prime} \in U$. We call $\approx_{\mathcal{W}}$ the global symmetry relation of $\mathcal{W}$. Let $[A]_{\approx_{\mathfrak{P}}}$ be the equivalence class of $A$ with respect to $\approx_{\mathcal{W}}$. It is easy to verify that $[A]_{\approx_{\mathfrak{P}}}$ is a unionclosed family (see [12] for details). Hence, the global symmetry relation for pairings yields specific models of families in the various mathematical contexts where pairings occur.

Now, since $[A]_{\approx_{\mathfrak{P}}}$ is a union closed family, it has a maximal element $M_{\mathcal{W}}(A)$, that we call the maximum partitioner of $A$. Then the set operator $M_{\mathcal{W}}: A \in \mathcal{P}(\Omega) \mapsto M_{\mathcal{W}}(A) \in \mathcal{P}(\Omega)$ is a closure operator on $\Omega$, the subset family

$$
\mathcal{M}(\mathcal{W}):=\left\{C \in \mathcal{P}(\Omega): M_{\mathcal{W}}(C)=C\right\}=\left\{M_{\mathcal{W}}(B): B \in \mathcal{P}(\Omega)\right\}
$$

is a closure system on $\Omega$ and $\mathbb{M}(\mathcal{W}):=\left(\mathcal{N}(\mathcal{W}), \subseteq^{*}\right)$ a complete lattice (see [12] for details). Moreover, given the preordered set $\mathbb{H}(\mathcal{W}):=\left(\mathcal{P}(\Omega), \geqslant_{\mathcal{W}}\right)$, where $A \geqslant \mathcal{W} A^{\prime}$ if $M_{\mathcal{W}}(A) \supseteq M_{\mathcal{W}}\left(A^{\prime}\right)$, it results that the global symmetry relation $\approx_{\mathcal{W}}$ coincides with the symmetrization of the preorder $\geqslant_{\mathcal{W}}$ and that $\mathbb{H}(\mathcal{W}) / \approx_{\mathcal{W}}$ is a lattice order-isomorphic to $\mathbb{M}(\mathcal{W})$ (see Proposition 14). This preorder satisfies the fundamental property

$$
X \geqslant_{\mathcal{W}} Y_{i}, \forall i \in I \Longrightarrow X \geqslant_{\mathcal{W}} \bigcup Y_{i}
$$

In view of Property (4), we will use the terminology union additive relation induced by $\mathcal{W}$ to indicate the preorder $\geqslant w$.

As a type of dual structure of $\mathcal{N}(\mathcal{W})$, the subset family $\mathcal{N}(\mathcal{W}):=\bigcup\left\{\min \left([A]_{\approx_{\mathfrak{P}}}\right)\right.$ : $A \in \mathcal{M}(\mathcal{W})\}$ has been introduced in [14] showing that it is an abstract simplicial complex that is related with $\mathcal{M}(\mathcal{W})$ by means of several properties and results. For example, for some types of pairings $\mathcal{M}(\mathcal{W})$ and $\mathcal{N}(\mathcal{W})$ are respectively the closed subset family and the independent subset family of a matroid (see [14] for details).

Based on recent results obtained in the graph context $[13,14]$ and on other general results linked to theoretical computer science $[1,2,12,15,25,26,36,37,39]$, in this paper we continue the study of the basic property of the set system $\mathcal{M}(\mathcal{W})$ and use methods from $\mathrm{GrC}$ in relation to order-theory, limited to finite lattices.

To this regard, we prove a representation result for the closure system $\mathcal{M}(\mathcal{W})$ and we insert it within a general research perspective involving the interrelations between combinatorics, order theory and topological structures. More in detail, we prove, in a constructive way, that the family of all closed sets of any closure system on some finite set $\Omega$ agrees with the family of the maximum partitioners of some pairing $\mathcal{W}$ on $\Omega$ itself (Theorem 5).

Through the previous result, we will provide two representation results concerning all the preorders on a set $\Omega$ satisfying (4) (which we call union additive relations on $\Omega$ ) and another one concerning finite lattices. On the one hand, we shall show that any union additive relation on $\Omega$ agrees with the preorder $\geqslant_{\mathcal{W}}$ induced by some pairing $\mathcal{W}$ on $\Omega$ itself. 
On the other hand, in order to provide a representation theorem for finite lattices in terms of pairings, we shall use a classical result of lattice theory, according to which any finite lattice can be represented as a closure system on the set of all join-irreducible elements of the lattice itself [6]. This is the keypoint of our first representation theorem, since we use the aforementioned result to show that for each finite lattice $\mathbb{L}$ there exists a pairing $\mathcal{W}_{\mathbb{L}}$ on some finite ground set $\Omega_{\mathbb{L}}$ such that the lattice $\mathbb{H}\left(\mathcal{W}_{\mathbb{L}}\right) / \approx_{\mathcal{W}_{\mathbb{L}}}$ is order-isomorphic to $\mathbb{L}$ itself (see Theorem 17). Therefore, as a consequence of such a representation theorem, we can reinterpret the order relation in any finite lattice $\mathbb{L}$ as the quotient of the preorder relation $\geqslant_{\mathcal{W}}$, for some $\mathcal{W}$ on $\Omega_{\mathbb{L}}$. In other terms, we can consider the order relation of any algebraic lattice as the quotient relation of a union additive relation defined on some set $\Omega_{\mathbb{L}}$. In this way, we provide an enrichment of the study of the lattice order theory establishing new interrelationships between order and topological properties by means of the union additive property given in (4).

Theorem 17 provides a refinement for the partial order $\leqslant_{\mathbb{L}}$ of the lattice. Indeed, setting

$$
\gamma_{\mathcal{W}}(A, B):=\frac{\left|\left\{u \in U:[u]_{A} \subseteq[u]_{B}\right\}\right|}{|U|}
$$

for each $A, B \in \mathcal{P}(\Omega)$ and fixing two elements of the lattice $x, y \in L$, it may be easily verified that $x \leqslant_{\mathbb{L}} y$ if and only if $\gamma_{\mathcal{W}}(\eta(x), \eta(y))=1$. This fact enables us to compute $\gamma_{\mathcal{W}}(\eta(x), \eta(y))$ even if $x$ and $y$ are non-comparable with respect to the order of the lattice $\mathbb{L}$. In other terms, the function $\gamma_{\mathcal{W}}$ provides extra numerical informations for partial dependencies of subsets, corresponding to two non-comparable nodes of the lattice $\mathbb{L}$ and that are not explicit in the lattice itself. Note that each node corresponds to an equivalence class with respect to the equivalence relation $\approx_{\mathcal{W}}$ and the value of $\gamma_{\mathcal{W}}$ corresponding to a pair of subsets belonging to the same equivalence class is clearly 1.

In general, given a pairing $\mathcal{W}$ on a finite set $\Omega$ with $n$ elements, it is always possible to put within a $2^{n} \times 2^{n}$ table $T(\mathcal{W})$ the values $\gamma_{\mathcal{W}}(X, Y)$. The complete determination of all the entries of the above table enables us to obtain the closure system $\mathcal{M}(\mathcal{W})$. In other terms, we may consider the table $T(\mathcal{W})$ as a sort of numerical completion of $\mathcal{M}(\mathcal{W})$. The map $\gamma_{\mathcal{W}}$ and some related averages have been broadly used in $[16,33]$ in order to investigate the transmission of symmetry in some basic digraph and graph families.

Eventually, in the last part of the paper we focus our attention on the link between a specific class of lattices endowed with an involutory map and some particular subset families induced by means of pairings. More in detail, we consider a finite lattice $\mathbb{L}$ endowed with an order-reversing involutory map $\psi: L \rightarrow L$ exchanging $\hat{0}_{\mathbb{L}}$ and $\hat{1}_{\mathbb{L}}$ and such that $\psi(\alpha) \wedge \alpha=\hat{0}_{\mathbb{L}}$ and $\psi(\alpha) \vee \alpha=\hat{1}_{\mathbb{L}}$. In this case we say that the pair $(\mathbb{L}, \psi)$ is a complemented involutory lattice. In general, the theory of the posets endowed of an involutory map is a well investigated research field $[4,11,24,32]$, which also has links with complex analysis [7] and design theory [9, 23] (moreover, for some studies concerning discrete dynamics on particular types of lattices endowed with involutory maps see $[8,10]$ ).

In the present context, given a pairing $\mathcal{W}$ on $\Omega$ and $A \in \mathcal{P}(\Omega)$, we set $K_{\mathcal{W}}(A):=\Omega \backslash$ $M_{\mathcal{W}}\left(A^{c}\right)$. Subsequently, we say that $A \in \mathcal{P}(\Omega)$ is normally extensible if $A=M_{\mathcal{W}}\left(K_{\mathcal{W}}(A)\right)$. Then we show that for any finite complemented involutory lattice $(\mathbb{L}, \psi)$, there exist a 
finite set $\Omega_{\mathbb{L}, \psi}$ and a pairing $\mathcal{W}$ on $\Omega_{\mathbb{L}, \psi}$ such that $M_{\mathcal{W}}(\emptyset)=\emptyset$ and for which the poset of all normally extensible subsets with respect to set theoretical inclusion is a complete lattice order-isomorphic to $\mathbb{L}$ (see Theorem 22).

Let us briefly describe the content of our paper. In Section 2 we first recall some basic notions and results on closure systems, lattices, families of subsets and pairings. In Section 3 we provide a constructive proof for the representation result for closure systems on finite sets (Theorem 5) and provide some examples of how our algorithm works. In Section 4 we introduce the notion of union additive relations and give the proof of the representation theorem for such relations. Furthermore, we will also provide a proof for the representation theorem of finite lattices, according to which for any finite lattice $\mathbb{L}$ we can find a finite set $\Omega_{\mathbb{L}}$ and a pairing $\mathcal{W}_{\mathbb{L}}$ on it such that $\mathbb{L}$ is order-isomorphic to $\mathcal{M}\left(\mathcal{W}_{\mathbb{L}}\right)$ (Theorem 17). Section 5 focuses the attention on the representation of complemented involutory lattices through a pairing $\mathcal{W}$ on a finite set $\Omega_{\mathbb{L}, \psi}$ such that the poset of all normally extensible subsets is order-isomorphic to $\mathbb{L}$ itself.

\section{Reviews, Notations and Basic Results}

Notations. In this paper we denote by $\Omega$ a given finite arbitrary set and by $\mathcal{P}(\Omega)$ the power set of $\Omega$. We use the symbol $\subseteq^{*}$ to denote dual inclusion, that is $A \subseteq \subseteq^{*} B$ is equivalent to say that $B \subseteq A$. If $n$ is a positive integer, we denote by $\Omega^{n}$ the cartesian product of $n$ copies of $\Omega$. When $X \subseteq \Omega$, we will use both the notations $X^{c}$ and $\Omega \backslash X$ to indicate the complement subset of $X$ with respect to $\Omega$. If $f: \Omega \rightarrow \Omega^{\prime}$ is a map between sets, we denote by $\operatorname{Im}(f)$ the image of $f$. A map $\psi$ is called an involutory map on $\Omega$ if $\psi^{2}=I d_{\Omega}$, where $I d_{\Omega}$ denotes the identity on $\Omega$.

If $X$ is a set and we have two maps $\psi: X \rightarrow X$ and $f: X \rightarrow \mathcal{P}(\Omega)$ such that $f(\psi(x))=\Omega \backslash f(x)$ for any $x \in X$, we say that $f$ is a $\psi$-complementary map.

Posets and Lattices. Let $\mathbb{P}:=(P, \leqslant)$ be a poset and $X \in \mathcal{P}(P)$ be a non-empty subset. If $x, y \in P$, we also write $x<y$ if $x \leqslant y$ and $x \neq y$. If $x, y$ are two distinct elements of $P$, we say that $y$ covers $x$, denoted by $x \lessdot y$ if $x \leqslant y$ and there exists no element $z \in P$ such that $x<z<y$.

Let $\mathbb{P}:=(P, \leqslant)$ and $\mathbb{P}^{\prime}:=\left(P^{\prime}, \leqslant^{\prime}\right)$ be two posets, $\alpha, \beta \in P$ and $f: P \rightarrow P^{\prime}$ a map. We say that $f$ is:

- order-preserving if $x \leqslant y \Longrightarrow f(x) \leqslant^{\prime} f(y)$ for each $x, y \in L$;

- order-reversing if $x \leqslant y \Longrightarrow f(y) \leqslant f(x)$ for each $x, y \in L$;

- $\{\alpha, \beta\}$-preserving if the restriction map $f_{\mid\{\alpha, \beta\}}:\{\alpha, \beta\} \rightarrow\{f(\alpha), f(\beta)\}$ is an order isomorphism.

Let $\mathbb{L}:=\left(L, \leqslant_{\mathbb{L}}\right)$ be a lattice. For each $x, y \in L$, we denote by $x \vee y$ the join and by $x \wedge y$ the meet of $x$ and $y$ in $\mathbb{L}$. When they exist, we denote by $\hat{0}_{\mathbb{L}}$ and $\hat{1}_{\mathbb{L}}$ the meet and the join of $L$, respectively. If $x \in \mathbb{L}$, we say that $x$ is join-irreducible if $x \neq \hat{0}_{\mathbb{L}}$ and $x=y \vee z$ implies $x=y$ or $x=z$ for any $y, z \in \mathbb{L}$. We denote by $\mathcal{J}(\mathbb{L})$ the subset of all 
join-irreducible elements of $\mathbb{L}$. A lattice $\mathbb{L}=(L, \leqslant \mathbb{L})$ is said complete if there exist both the join and the meet of any subset $X \in \mathcal{P}(L)$ and we denote them by $\bigvee X$ and $\bigwedge X$, respectively.

We call a pair $(\mathbb{L}, \psi)$ a complemented involutory lattice if $\psi: L \rightarrow L$ is an orderreversing involutory map such that $\psi\left(\hat{0}_{\mathbb{L}}\right)=\hat{1}_{\mathbb{L}}, \psi\left(\hat{1}_{\mathbb{L}}\right)=\hat{0}_{\mathbb{L}}$ and, for any $\alpha \in L$, it results $\psi(\alpha) \wedge \alpha=\hat{0}_{\mathbb{L}}$ and $\psi(\alpha) \vee \alpha=\hat{1}_{\mathbb{L}}$.

The most simple example of a complemented involutory lattice is the poset $(\mathcal{P}(\Omega), \subseteq)$ where $\psi: \mathcal{P}(\Omega) \rightarrow \mathcal{P}(\Omega)$ associates with each set its complement. More in general, whenever we fix a set system $\mathcal{F} \in \mathcal{P}(\mathcal{P}(\Omega))$ and consider the lattice $\mathbb{L}_{\mathcal{F}}:=(\mathcal{X}, \subseteq)$, where $X$ consists of $\emptyset, \Omega$, the members of $\mathcal{F}$ and their complements, we obtain a complemented involutory lattice by taking the map $\psi$ associating with each set of $\mathcal{F}$ its complement and exchanging $\emptyset$ with $\Omega$.

We denote by $M_{n}$ the linear sum $\mathbf{1} \oplus \overline{\mathbf{n}} \oplus \mathbf{1}$ (see page 17 of [19] for details), where $\overline{\mathbf{n}}$ is the antichain on $n$ elements $\{1, \ldots, n\}$. Another simple example of a complemented involutory lattice is given by $\left(M_{n}, \psi\right)$, with $n$ an even integer, $\psi\left(a_{i}\right)=a_{\sigma(i)}$, where $\sigma$ is a product of disjoint transpositions without fixed elements, $\psi\left(\hat{0}_{\mathbb{L}}\right)=\hat{1}_{\mathbb{L}}$ and $\psi\left(\hat{1}_{\mathbb{L}}\right)=\hat{0}_{\mathbb{L}}$.

Set Systems and Set Operators. We call the elements of $\mathcal{P}(\mathcal{P}(\Omega))$ set systems on $\Omega$. A set system $\mathcal{F}$ on $\Omega$ is said:

- union-closed if whenever $\mathcal{F}^{\prime} \subseteq \mathcal{F}$ then $\cup \mathcal{F}^{\prime} \in \mathcal{F}$;

- complement-closed if $\Omega \in \mathcal{F}$ and $Z \in \mathcal{F} \Longrightarrow Z^{c} \in \mathcal{F}$;

- a closure system on $\Omega$ if $\Omega \in \mathcal{F}$ and $\cap \mathcal{F}^{\prime} \in \mathcal{F}$ whenever $\mathcal{F}^{\prime} \subseteq \mathcal{F}$.

We recall that if $\mathcal{F}$ is a closure system on $\Omega$, then the poset $(\mathcal{F}, \subseteq)$ is a complete lattice (usually called closure lattice of $\mathcal{F}$ ) in which the meet operation is the subset intersection.

A set operator on $\Omega$ is any map $\sigma: \mathcal{P}(\Omega) \rightarrow \mathcal{P}(\Omega)$. For any set operator $\sigma$ on $\Omega$, the fixed point set of $\sigma$ is the following set system of $\Omega$ :

$$
\operatorname{Fix}(\sigma):=\{A \in \mathcal{P}(\Omega): \sigma(A)=A\}
$$

Moreover, if $\mathcal{F}$ is a given set system on $\Omega$ we will consider the induced set operator Int $_{\mathcal{F}}$ on $\Omega$ defined by

$$
\operatorname{Int}_{\mathcal{F}}(A):=\bigcap\{B \in \mathcal{F}: A \subseteq B\}
$$

A set operator $\sigma$ on $\Omega$ is said:

- isotone, if whenever $A, B \in \mathcal{P}(\Omega)$ and $A \subseteq B$, then $\sigma(A) \subseteq \sigma(B)$;

- extensive, if $A \subseteq \sigma(A)$ for all $A \in \mathcal{P}(\Omega)$;

- intensive, if $\sigma(A) \subseteq A$ for all $A \in \mathcal{P}(\Omega)$;

- idempotent, if $\sigma(\sigma(A))=\sigma(A)$ for all $A \in \mathcal{P}(\Omega)$;

- a closure operator, if $\sigma$ is isotone, extensive and idempotent; 
- a kernel operator, (see [22]) if $\sigma$ is isotone, intensive and idempotent.

In the next theorem, we recall the well-known bijective correspondence between closure systems and closure operators.

Theorem 1 (Thm 7.3, [19]). Let $\sigma$ be a closure operator on $\Omega$ and $\mathcal{F}$ be a closure system on $\Omega$. Then Fix $(\sigma)$ is a closure system on $\Omega$. and Int I $_{\mathcal{F}}$ is a closure operator on $\Omega$. Moreover, we have that

$$
\operatorname{Int} t_{F i x(\sigma)}=\sigma, \quad F i x\left(\operatorname{Int}_{\mathcal{F}}\right)=\mathcal{F}
$$

The next result, whose proof is straightforward, will be useful in what follows.

Proposition 2. Let $\mathcal{F}$ be a complement-closed set system on $\Omega$. Then Int $_{\mathcal{F}}$ is a closure operator on $\Omega$ such that $\operatorname{Int}_{\mathcal{F}}(\emptyset)=\emptyset$.

Set Partitions. If $\pi$ is a set partition on $\Omega$, we usually denote by $\left\{B_{i}: i \in I\right\}$ the block family of $\pi$. If $u \in \Omega$, we denote by $\pi(u)$ the block of $\pi$ which contains the element $u$. When $\Omega$ is finite we use the standard notation $\pi:=B_{1}|\ldots| B_{|\pi|}$, where $|\pi|$ is the number of distinct blocks of $\pi$. We denote by $\Pi(\Omega)$ the set of all set-partitions of $\Omega$. It is well-known that on the set $\Pi(\Omega)$ we can consider a partial order $\preceq$ defined as follows: if $\pi, \pi^{\prime} \in \Pi(\Omega)$, then

$$
\pi \preceq \pi^{\prime}: \Longleftrightarrow(\forall B \in \pi)\left(\exists B^{\prime} \in \pi^{\prime}\right): B \subseteq B^{\prime} \Longleftrightarrow(\forall u \in \Omega)\left(\pi(u) \subseteq \pi^{\prime}(u)\right) .
$$

We will write $\pi \prec \pi^{\prime}$ when $\pi \preceq \pi^{\prime}$ and $\pi \neq \pi^{\prime}$. The pair $(\Pi(\Omega), \preceq)$ is a complete lattice which is called partition lattice of the set $\Omega$.

Pairings. We call a quadruple $\mathcal{W}:=\langle F, U, \Omega, \Lambda\rangle$, where $U$ and $\Omega$ are finite non-empty sets, $\Lambda$ is a non-empty set and $F$ is a map having domain $U \times \Omega$ and codomain $\Lambda$, a pairing on $\Omega$.

For each $A \in \mathcal{P}(\Omega)$ we consider the following equivalence relation $\equiv_{A}$ on $U$ :

$$
u \equiv_{A} u^{\prime}: \Longleftrightarrow F(u, a)=F\left(u^{\prime}, a\right) \forall a \in A .
$$

for any $u, u^{\prime} \in U$. Let $[u]_{A}$ be the equivalence class of $u$ with respect to $\equiv_{A}$ and

$$
\pi_{\mathcal{W}}(A):=\left\{[u]_{A}: u \in U\right\}
$$

the set partition on $U$ induced by $\equiv_{A}$. Let us note that, for any $A^{\prime} \in \mathcal{P}(\Omega)$, we have that

$$
A \subseteq A^{\prime} \Longrightarrow \pi_{\mathcal{W}}\left(A^{\prime}\right) \preceq \pi_{\mathcal{W}}(A)
$$

We set

$$
\Pi_{\text {sym }}(\mathcal{W}):=\left\{\pi_{\mathcal{W}}(B): B \in \mathcal{P}(\Omega)\right\}, \mathbb{P}_{\text {sym }}(\mathcal{W}):=\left(\Pi_{\text {sym }}(\mathcal{W}), \preceq\right),
$$

so that $\mathbb{P}_{\text {sym }}(\mathcal{W})$ is a sub-poset of the partition lattice $(\Pi(U), \preceq)$. 
In what follows, we will consider the equivalence relation $\approx_{\mathcal{W}}$ on the power set $\mathcal{P}(\Omega)$ defined by

$$
A \approx_{\mathcal{W}} A^{\prime}: \Longleftrightarrow \pi_{\mathcal{W}}(A)=\pi_{\mathcal{W}}\left(A^{\prime}\right),
$$

for any $A, A^{\prime} \in \mathcal{P}(\Omega)$, and we will denote by $[A]_{\approx_{\mathfrak{P}}}$ the equivalence class of $A$ with respect to $\approx$ w.

The basic properties of the relation $\approx_{\mathcal{W}}$ are recalled in the following result.

Theorem 3 (Proposition 3.2, [12]). We have that:

(i) If $A \approx_{\mathcal{W}} A^{\prime}$ and $D \in \mathcal{P}(\Omega)$, then $A \cup D \approx_{\mathcal{W}} A^{\prime} \cup D$.

(ii) The equivalence class $[A]_{\approx_{\mathcal{W}}}$ is union-closed and, hence, it has only a maximum element $\bigcup[A]_{\approx_{\mathcal{W}}}$, which we denote by $M_{\mathcal{W}}(A)$.

(iii) $M_{\mathcal{W}}(A)=\left\{b \in \Omega: A \cup\{b\} \approx_{\mathcal{W}} A\right\}=\left\{a \in \Omega:\left(u, u^{\prime} \in \Omega \wedge u \equiv_{A} u^{\prime}\right) \Longrightarrow F(u, a)=\right.$ $\left.F\left(u^{\prime}, a\right)\right\}$.

(iv) $M_{\mathcal{W}}(A) \supseteq M_{\mathcal{W}}\left(A^{\prime}\right) \Longleftrightarrow \pi_{\mathcal{W}}(A) \preceq \pi_{\mathcal{W}}\left(A^{\prime}\right)$.

(v) The set operator $M_{\mathcal{W}}: B \in \mathcal{P}(\Omega) \mapsto M_{\mathcal{W}}(B) \in \mathcal{P}(\Omega)$ is a closure operator on $\Omega$, whose induced closure system is

$$
\mathcal{M}(\mathcal{W}):=\left\{M_{\mathcal{W}}(B): B \in \mathcal{P}(\Omega)\right\}=\left\{C \in \mathcal{P}(\Omega): M_{\mathcal{W}}(C)=C\right\}
$$

Moreover, the poset $\mathbb{M}(\mathcal{W}):=\left(\mathcal{M}(\mathcal{W}), \subseteq^{*}\right)$ is a complete lattice such that if $\left\{A_{j}: j \in\right.$ $J\} \subseteq \mathcal{M}(\mathcal{W})$ then

$$
\bigvee_{j \in J} A_{j}=\bigcap_{j \in J} A_{j} \text { and } \bigwedge_{j \in J} A_{j}=M_{\mathcal{W}}\left(\bigcup_{j \in J} A_{j}\right)
$$

Finally, the map $\pi_{\mathfrak{P}}: A \in \mathcal{N}(\mathcal{W}) \mapsto \pi_{\mathcal{W}}(A) \in \Pi_{\text {sym }}(\mathcal{W})$ induces an order isomorphism between the closure lattice $\mathbb{M}(\mathcal{W})$ and the poset $\mathbb{P}_{\text {sym }}(\mathcal{W})$. In particular, $\mathbb{P}_{\text {sym }}(\mathcal{W})$ is a lattice.

In what follows, we call the elements of $\mathcal{M}(\mathcal{W})$ the maximum partitioners of $\mathcal{W}$, the lattice $\mathbb{M}(\mathcal{W})$ the maximum partitioner lattice of $\mathcal{W}$ and $\mathbb{P}_{\text {sym }}(\mathcal{W})$ the symmetry partition lattice of $\mathcal{W}$.

Remark 4. There is another lattice which is order isomorphic to the dual closure lattice $\mathbb{M}(\mathcal{W})$, whose role is relevant when ones uses micro and macro granular representations induced by information tables (see [14] for details). Such a lattice is obtained by taking firstly the set $\mathcal{G}(\mathcal{W}):=\left\{[A]_{\approx \mathcal{W}}: A \in \mathcal{M}(\mathcal{W})\right\}$, and next by considering the partial order $\sqsubseteq$ on $\mathcal{G}(\mathcal{W})$ defined by: $[A]_{\approx_{\mathcal{W}}} \sqsubseteq\left[A^{\prime}\right]_{\approx_{\mathcal{W}}}: \Longleftrightarrow A \subseteq^{*} A^{\prime}$, for any $[A]_{\approx_{\mathcal{W}}},\left[A^{\prime}\right]_{\approx_{\mathcal{W}}} \in \mathcal{G}(\mathcal{W})$. Then, by Theorem 3 one can deduce that the poset $\mathbb{G}(\mathcal{W}):=(\mathcal{G}(\mathcal{W})$, $)$ is a lattice which is order-isomorphic to the maximum partitioner lattice $\mathbb{M}(\mathcal{W})$ (see [12] for details). Relatively to a specific pairing, we shall visualize the lattice $\mathbb{G}(\mathcal{W})$ in Example 7.

\section{Representation Theorem for Closure Systems}

The main result of the present section is Theorem 5 , which is a representation result for closure systems on $\Omega$ by means of pairings on the same ground set. More specifically, for 
any closure system $\mathcal{S}$ on $\Omega$ we are able to find a pairing $\mathcal{W}$ on $\Omega$ such that the set system $\mathcal{M}(\mathcal{W})$ of all maximum partitioners of $\mathcal{W}$ coincides with $\mathcal{S}$.

The proof of Theorem 5 is constructive and it is based on an algorithmic construction whose underlying idea derives by the simplest case when a closure system is a chain of subsets, as in Example 6.

The main idea of such a proof is based on the fact that the order structure of the given closure lattice on $\Omega$ can be entirely described by its maximal chains.

Theorem 5. The map associating with any pairing $\mathcal{W}$ on $\Omega$ the closure system $\mathcal{M}(\mathcal{W})$ on $\Omega$ is surjective.

Proof. Let $\mathcal{S}$ be a closure system on $\Omega=\left\{a_{1}, \ldots, a_{n}\right\}$ and $\mathbb{S}:=(\mathcal{S}, \subseteq)$ its induced closure lattice. Then $\mathbb{S}$ has maximum element $\hat{1}_{\mathbb{S}}=\Omega$ and minimum element $\hat{0}_{\mathbb{S}}:=E=\cap \mathcal{S}$. We denote by $\lessdot$ the covering relation of $\mathbb{S}$.

Let $\mathcal{C}_{1}, \mathcal{C}_{2}, \ldots, \mathcal{C}_{k}$ be all the maximal chains of $\mathbb{S}$. Obviously, any chain $\mathcal{C}_{i}$ has bottom $E$ and top $\Omega$.

We use the following notations for the previous chains:

$$
\begin{gathered}
\mathcal{C}_{1}: A_{1,1}=E \lessdot A_{1,2} \lessdot \cdots \lessdot A_{1, l_{1}}=\Omega, \\
\vdots \\
\mathcal{C}_{k}: A_{k, 1}=E \lessdot A_{k, 2} \lessdot \cdots \lessdot A_{k, l_{k}}=\Omega,
\end{gathered}
$$

so that the positive integer $l_{i}$ agrees with the length of the chain $\mathcal{C}_{i}$, for any $i=1, \ldots, k$.

Set

$$
\begin{aligned}
m_{0}:=0, m_{1}:=l_{1}, m_{2}: & =l_{1}+l_{2}, \ldots, m_{k}:=l_{1}+\cdots+l_{k}, \\
m & :=m_{k},
\end{aligned}
$$

and

$$
U_{\mathcal{S}}:=\{1,2, \ldots, m\}
$$

Let us note that we have the following partition of $U_{\mathcal{S}}$ with integer intervals:

$$
\left[m_{0}+1, m_{1}\right],\left[m_{1}+1, m_{2}\right], \ldots,\left[m_{k-1}+1, m_{k}\right]
$$

Therefore, for any element $u \in U_{\mathcal{S}}$ there exists a unique index $\iota_{u} \in\{1,2, \ldots, k\}$ such that

$$
u \in\left[m_{\iota_{u}-1}+1, m_{\iota_{u}}\right]
$$

Now we will construct a pairing

$$
\mathcal{W}=\mathcal{W}(\mathcal{S}):=\left\langle F_{\mathcal{S}}, U_{\mathcal{S}}, \Omega, \mathbb{N}\right\rangle
$$

on $\Omega$ for which $\mathcal{M}(\mathcal{W})=\mathcal{S}$.

To this purpose, we will define recursively the map $F_{\mathcal{S}}: U_{\mathcal{S}} \times \Omega \longrightarrow \mathbb{N}$. 
Firstly, let us note that the greatest element of any maximal chain is the whole set $\Omega$, therefore for any $1 \leqslant i \leqslant k$ and any $a \in \Omega$ there exists the following minimum integer number:

$$
\zeta_{i, a}:=\min \left\{q \in\left\{1,2, \ldots, l_{i}\right\}: a \in A_{i, q}\right\}
$$

Now we associate with any ordered pair $(u, a) \in U_{\mathcal{S}} \times \Omega$ the following integer set:

$$
J_{u, a}:= \begin{cases}{\left[m_{\iota_{u}-1}+2, m_{\iota_{u}}-\zeta_{\iota_{u}, a}+1\right]} & \text { if } m_{\iota_{u}-1}+2 \leqslant m_{\iota_{u}}-\zeta_{\iota_{u}, a}+1 \\ \emptyset & \text { otherwise }\end{cases}
$$

At this point we define $F_{\mathcal{S}}$ recursively, as follows.

Firstly, for any $a \in E$, we set

$$
F_{\mathcal{S}}(u, a):=1
$$

for all $u \in U$.

On the other hand, for any $a \in \Omega \backslash E$, we set

$$
F_{\mathcal{S}}(1, a):=1
$$

and

$$
F_{\mathcal{S}}(u, a):= \begin{cases}F_{\mathcal{S}}(u-1, a) & \text { if } u \in J_{u, a} \\ F_{\mathcal{S}}(u-1, a)+1 & \text { if } u \notin J_{u, a}\end{cases}
$$

for all $u=2, \ldots, m$.

Let us consider the pairing $\mathcal{W}=\left\langle F_{\mathcal{S}}, U_{\mathcal{S}}, \Omega, \mathbb{N}\right\rangle$ so constructed. We shall demonstrate that $\mathcal{M}(\mathcal{W})=\mathcal{S}$.

For, let us firstly show the inclusion $\mathcal{S} \subseteq \mathcal{M}(\mathcal{W})$. Let $A \in \mathcal{S}$. We distinguish three distinct cases.

(i): Let first $A=\Omega$. In this case $\Omega \in \mathcal{M}(\mathcal{W})$ because $\mathcal{M}(\mathcal{W})$ is a closure system on $\Omega$.

(ii): Let $A=E$. In such a case, it suffices to see that $E=M_{\mathcal{W}}(\emptyset)$.

In fact, by definition of $F_{\mathcal{S}}$ we have that $\pi_{\mathcal{W}}(E)=U_{\mathcal{S}}=\pi_{\mathcal{W}}(\emptyset)$, therefore $E \in[\emptyset]_{\approx \mathcal{W}}$, hence $E \subseteq M_{\mathcal{W}}(\emptyset)$, because $M_{\mathcal{W}}(\emptyset)$ is the maximum element of $[\emptyset]_{\approx_{\mathcal{W}}}$.

Conversely, let $a \in M_{\mathcal{W}}(\emptyset)$. Then, in view (iii) of Theorem 3, we have that

$$
F_{\mathcal{S}}(u, a)=F_{\mathcal{S}}\left(u^{\prime}, a\right) \forall u, u^{\prime} \in U_{\mathcal{S}}
$$

Let us assume by contradiction that $a \notin E$, and we take $v=m_{1} \in U_{\mathcal{s}}$, so that $\iota_{v}=1$. Therefore

$$
J_{v, a}=\left[2, m_{1}+1-\zeta_{1, a}\right]
$$

Then, it results that $v \notin J_{v, a}$ since $\zeta_{1, a} \geqslant 2$. So, by $(10)$ we have that $F_{\mathcal{S}}(v, a) \neq F_{\mathcal{S}}(v-1, a)$, and this contradicts (11). On the other hand, the inclusion $M_{\mathcal{W}}(\emptyset) \subseteq E$ also holds. Hence we get $E=M_{\mathcal{W}}(\emptyset) \in \mathcal{M}(\mathcal{W})$.

(iii): Let now $E \varsubsetneqq A \varsubsetneqq \Omega$. In view of part (iii) of Theorem 3, it suffices to prove the existence of two elements $w, w^{\prime}$ of $U_{\mathcal{S}}$ such that

$$
w \equiv_{A} w^{\prime} \text { and } F_{\mathcal{S}}(w, b) \neq F_{\mathcal{S}}\left(w^{\prime}, b\right) \forall b \in \Omega \backslash A
$$


In fact, if the condition (12) is satisfied for some $w, w^{\prime} \in U_{\mathcal{S}}$, by (iii) of Theorem 3 we have that $M_{\mathcal{W}}(A) \subseteq A$, therefore $A=M_{\mathcal{W}}(A) \in \mathcal{M}(\mathcal{W})$.

Then, in order to find two specific elements $w, w^{\prime} \in U$ that satisfy the condition (12), we proceed as follows.

Since $E \varsubsetneqq A \varsubsetneqq \Omega$, it follows that any maximal chain of $\mathbb{S}$ has length at least three. Let then

$$
\mathcal{C}_{i}: A_{i, 1}=E \lessdot A_{i, 2} \lessdot \cdots \lessdot A_{i, l_{i}}=\Omega
$$

be a given maximal chain of $\mathbb{S}$ such that $A=A_{i, t}$, for some $t \in\left\{2, \ldots, l_{i}-1\right\}$. Set $w:=m_{i}-t$ and $w^{\prime}:=w+1$. Let us firstly show that $w \equiv_{A} w^{\prime}$. Indeed, if $a \in E$, then $F_{\mathcal{S}}(w, a)=F_{\mathcal{S}}\left(w^{\prime}, a\right)=F_{\mathcal{S}}(w+1, a)=1$ in view of the definition of $F_{\mathcal{S}}$; while, if $a \in A \backslash E$, we get $\zeta_{i, a} \leqslant t$ because of the definition of $\zeta_{i, a}$. Thus, by the latter condition and by the definition of both $t$ and $w$, we get $m_{i-1}+1 \leqslant w \leqslant m_{i}-\zeta_{i, a}$. In view of (10), since $m_{i-1}+2 \leqslant w^{\prime}=w+1 \leqslant m_{i}-\zeta_{i, a}+1$, it follows that $F_{\mathcal{S}}\left(w^{\prime}, a\right)=F_{\mathcal{S}}(w, a)$. Therefore, we have $w \equiv_{A} w^{\prime}$.

At this point, we shall demonstrate that for each choice of $b \in \Omega \backslash A$, it holds $F_{\mathcal{S}}(w, b) \neq$ $F_{\mathcal{S}}\left(w^{\prime}, b\right)$. First of all, notice that if $b \notin A$, then $b \notin E$ since $E \varsubsetneqq A$. Moreover, the definition of $\zeta_{i, b}$ implies that $\zeta_{i, b}>t$, whence $w=m_{i}-t>m_{i}-\zeta_{i, b}$ and $w^{\prime}=w+1>$ $m_{i}-\zeta_{i, b}+1$. Thus, again by (10), it follows that $F_{\mathcal{S}}\left(w^{\prime}, b\right)=F_{\mathcal{S}}(w+1, b)=F_{\mathcal{S}}(w, b)+1 \neq$ $F_{\mathcal{S}}(w, b)$, i.e. $w \not_{A} w^{\prime}$. This proves (12) and, hence, we conclude that $A \in \mathcal{M}(\mathcal{W})$.

It remains to prove the inclusion $\mathcal{M}(\mathcal{W}) \subseteq \mathcal{S}$. For, let $A \in \mathcal{M}(\mathcal{W})$. We claim that $A \in \mathcal{S}$. As above, we may distinguish three cases.

$\left(i^{\prime}\right)$ : If $A=M_{\mathcal{W}}(\emptyset)$, in view of the fact that $M_{\mathcal{W}}(\emptyset)=E$, we get $A \in \mathcal{S}$.

$\left(i i^{\prime}\right)$ : Analogously, if $A=\Omega$, then we get $A \in \mathcal{S}$ in view of the definition of a closure system.

$\left(i i i^{\prime}\right)$ : Thus, let $E \varsubsetneqq A \varsubsetneqq \Omega$. Let us consider the set system

$$
\mathcal{S}_{A}:=\{C \in \mathcal{S}: A \subseteq C\} .
$$

Let us note that $\mathcal{S}_{A}$ is again a closure system on $\Omega$, because $\Omega \in \mathcal{S}_{A}$ and $\mathcal{S}_{A}$ is intersection closed. Therefore there exists a minimum element $B \in \mathcal{S}_{A}$.

At this point, in order to prove that $A \in \mathcal{S}$, it suffices to show that $B \subseteq A$, since in such a case we will get $A=B \in \mathcal{S}$.

Let therefore $b \in B$. We must prove that $b \in A$, and this is equivalent to show that

$$
b \in M_{\mathcal{W}}(A)=A,
$$

because $A \in \mathcal{M}(\mathcal{W})$. Moreover, by part (iii) of Theorem 3, the condition $b \in M_{\mathcal{W}}(A)$ is equivalent to the following:

$$
\forall u, u^{\prime} \in U_{\mathcal{S}}, u \equiv_{A} u^{\prime} \Longrightarrow F_{\mathcal{S}}(u, b)=F_{\mathcal{S}}\left(u^{\prime}, b\right)
$$

Let us show that (13) is equivalent to the following:

$$
\forall u \in U_{\mathcal{S}}, u \equiv_{A} u-1 \Longrightarrow F_{\mathcal{S}}(u, b)=F_{\mathcal{S}}(u-1, b) .
$$


To see that, it suffices to show that (14) implies (13), since the reverse implication is obvious. To this regard, let $b \in M_{\mathcal{W}}(A)$ and $u, u^{\prime} \in U_{\mathcal{S}}$ be such that $u \equiv_{A} u^{\prime}$. We claim that $F_{\mathcal{S}}(u, b)=F_{\mathcal{S}}\left(u^{\prime}, b\right)$. Without loss of generality, we may assume $u^{\prime}<u$. In view of (10), the map $F_{\mathcal{S}}(\cdot, a)$ is non-decreasing. Therefore, the condition $u \equiv_{A} u^{\prime}$, or equivalently, $F_{\mathcal{S}}(u, a)=F_{\mathcal{S}}\left(u^{\prime}, a\right)$ for each $a \in A$ ensures that $F_{\mathcal{S}}(u, a)=F_{\mathcal{S}}(u-1, a)$ for each $a \in A$, i.e. $u \equiv_{A} u-1$. Hence, $F_{\mathcal{S}}(u, b)=F_{\mathcal{S}}(u-1, b)$. In particular, we also have $u^{\prime} \equiv_{A} u-1$, therefore we may proceed as before to show that $F_{\mathcal{S}}(u)=F_{\mathcal{S}}(u-1, b)=F_{\mathcal{S}}(u-2, b)$. Thus, iterating the previous argument, we get $F_{\mathcal{S}}(u, b)=F_{\mathcal{S}}\left(u^{\prime}, b\right)$. This shows the equivalence between (13) and (14).

Therefore, in order to complete the proof of the theorem, fix an arbitrary element of $u \in U_{\mathcal{S}}$ such that $u \equiv_{A} u-1$. We shall prove that

$$
F_{\mathcal{S}}(u, b)=F_{\mathcal{S}}(u-1, b)
$$

For each maximal chain $\mathcal{C}_{i}$, with $1 \leqslant i \leqslant k$, let us consider the set system

$$
\mathcal{C}_{i, A}:=\left\{A_{i, j} \in \mathcal{C}_{i}: A \subseteq A_{i, j}\right\}
$$

Clearly, $\Omega \in \mathcal{C}_{i, A}$; thus, there exists a minimum element in the chain $\mathcal{C}_{i}$ containing $A$. In other terms, for each $i=1, \ldots, k$, there exists a minimal integer $s_{i}$ such that

$$
2 \leqslant s_{i} \leqslant l_{i} \text { and } A \subseteq A_{i, s_{i}}
$$

In view of the minimality of $B$, we infer that $B \subseteq A_{i, s_{i}}$ for each $i=1, \ldots, k$, whence

$$
b \in B \subseteq \bigcap_{i=1}^{k} A_{i, s_{i}}
$$

Now, let us observe that

$$
\{u-1, u\} \subseteq\left[m_{\iota_{u}-1}+1, m_{\iota_{u}}\right]
$$

In fact, assume by contradiction that (17) does not hold. In such a case, by (7) we must have necessarily $u=m_{\iota_{u}-1}+1$. Moreover, since $E \varsubsetneqq A$, we can choose an element $\hat{a} \in A \backslash E$. Then, relatively to such a choice, by (9) it results that $u \notin J_{u, \hat{a}}$. Therefore, by (10) we obtain that

$$
F_{\mathcal{S}}(u, \hat{a}):=F_{\mathcal{S}}(u-1, \hat{a})+1 \neq F_{\mathcal{S}}(u-1, \hat{a}),
$$

and this is in contrast with the hypothesis $u \equiv_{A} u-1$. Hence (17) holds.

At this point, we can prove (15).

In view of (10), it suffices to show that

$$
u \in J_{u, b},
$$

that is

$$
m_{\iota_{u}-1}+2 \leqslant u \leqslant m_{\iota_{u}}-\zeta_{\iota_{u}, b}+1
$$


Now, the first of the previous inequality follows immediately by (17), since $m_{\iota_{u}-1}+1 \leqslant$ $u-1$. Therefore, in order to conclude our proof, it remains to show that

$$
u \leqslant m_{\iota_{u}}-\zeta_{\iota_{u}, b}+1
$$

Let $\bar{a} \in A \backslash E$ such that

$$
\zeta_{\iota_{u}, \bar{a}}:=\max \left\{\zeta_{\iota_{u}, a}: a \in A \backslash E\right\}
$$

Then we have that

$$
\zeta_{\iota u, \bar{a}}=s_{\iota u}
$$

In fact, since $A \subseteq A_{\iota_{u}, s_{\iota_{u}}}$, in view of the definition of the integer $\zeta_{\iota_{u}, \bar{a}}$, we must necessarily have $\zeta_{\iota_{u}, \bar{a}} \leqslant s_{\iota_{u}}$. Furthermore, if it were $\zeta_{\iota_{u}, \bar{a}}<s_{\iota_{u}}$, then we infer that

$$
A \subseteq A_{\iota_{u}, t} \varsubsetneqq A_{\iota_{u}, s_{\iota u}}
$$

where $A_{\iota_{u}, t} \in \mathcal{C}_{\iota_{u}}$, and this contradicts the minimality of the integer $s_{\iota_{u}}$. Therefore, (19) holds.

Now, since $u \equiv_{A} u-1$, it results that $F_{\mathcal{S}}(u, \bar{a})=F_{\mathcal{S}}(u-1, \bar{a})$. By (10), this implies that $u \in J_{u, \bar{a}}$. Therefore, by (9) and (19) we have that

$$
u \leqslant m_{\iota_{u}}-\zeta_{\iota_{u}, \bar{a}}+1=m_{\iota_{u}}-s_{\iota_{u}}+1 .
$$

On the other hand, by (16) we have that $b \in B \subseteq A_{\iota_{u}, s_{\iota_{u}}}$, and hence

$$
\zeta_{\iota_{u}, b} \leqslant s_{\iota_{u}}
$$

by $(8)$.

Finally, we obtain (18) as a direct consequence of (20) and (21).

Example 6. Let us consider the set $\Omega_{4}=\left\{a_{1}, a_{2}, a_{3}, a_{4}\right\}$ and the closure system

$$
\mathcal{S}=\left\{\left\{a_{1}\right\},\left\{a_{1}, a_{2}\right\},\left\{a_{1}, a_{2}, a_{3}\right\}, \Omega\right\}
$$

The closure $\mathbb{S}$ has the Hasse diagram represented in Figure 1, therefore $\mathbb{S}$ is a chain of length four.

The minimum in $\mathbb{S}$ is $E=\left\{a_{1}\right\}$, and in such a case we have only a maximal chain $\mathcal{C}_{1}$, which is $\mathbb{S}$ itself. Therefore, by using the same notations adopted in the proof of Theorem 5, we have that

$$
\mathcal{C}_{1}: E=A_{1,1}=\left\{a_{1}\right\} \lessdot A_{1,2}=\left\{a_{1}, a_{2}\right\} \lessdot A_{1,3}=\left\{a_{1}, a_{2}, a_{3}\right\} \lessdot A_{1,4}=\Omega_{4},
$$

$m_{0}=0, m=m_{1}=4$ and

$$
U=\left\{u_{1}:=1, u_{2}:=2, u_{3}:=3, u_{4}:=4\right\}
$$

Therefore

$$
\iota_{1}=\iota_{2}=\iota_{3}=\iota_{4}=1
$$




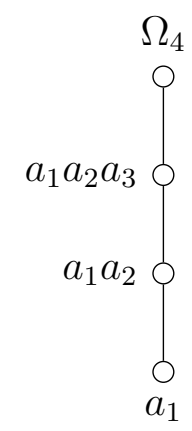

Figure 1: The Hasse Diagram of the chain with 4 elements.

\begin{tabular}{|l|l|l|l|l|}
\hline & $a_{1}$ & $a_{2}$ & $a_{3}$ & $a_{4}$ \\
\hline$u_{1}$ & 1 & $1 \downarrow$ & $1 \downarrow$ & $1 \downarrow$ \\
\hline$u_{2}$ & 1 & $u_{2} \in[2,3] \rightarrow 1 \downarrow$ & $u_{2} \in[2,2] \rightarrow 1 \downarrow$ & $u_{2} \notin \emptyset \rightarrow 1+1 \downarrow$ \\
\hline$u_{3}$ & 1 & $u_{3} \in[2,3] \rightarrow 1 \downarrow$ & $u_{3} \notin[2,2] \rightarrow 1+1 \downarrow$ & $u_{3} \notin \emptyset \rightarrow 2+1 \downarrow$ \\
\hline$u_{4}$ & 1 & $u_{4} \notin[2,3] \rightarrow 1+1$ & $u_{4} \notin[2,2] \rightarrow 2+1$ & $u_{4} \notin \emptyset \rightarrow 3+1$ \\
\hline
\end{tabular}

Figure 2: A visualization of the recursive construction of the map $F_{\delta}$ for the chain with 4 elements.

and

$$
\zeta_{1, a_{1}}=1, \zeta_{1, a_{2}}=2, \zeta_{1, a_{3}}=3, \zeta_{1, a_{4}}=4
$$

Now, in the table represented in Figure 2, we show as acts the algorithm which defines the map $F_{\mathcal{S}}$ in the proof of Theorem 5. We start from the top of any column $a_{j} \notin E$ and we move towards below. When $u_{i} \in J_{u_{i}, a_{j}}$ then we do not change the previous value, otherwise we sum 1 to the above value. We proceed in such a way for any column $a_{j} \notin E$. To the end of this process we obtain the pairing represented in Figure 3.

Example 7. Let us consider $\Omega_{5}:=\left\{a_{1}, a_{2}, a_{3}, a_{4}, a_{5}\right\}$ and the following closure system on $\Omega_{5}$ :

$$
\mathcal{S}:=\left\{\left\{a_{2}\right\},\left\{a_{2}, a_{5}\right\},\left\{a_{2}, a_{3}\right\},\left\{a_{2}, a_{3}, a_{4}\right\},\left\{a_{1}, a_{2}, a_{3}\right\}, \Omega_{5}\right\}
$$

\begin{tabular}{|l|l|l|l|l|}
\hline & $a_{1}$ & $a_{2}$ & $a_{3}$ & $a_{4}$ \\
\hline$u_{1}$ & 1 & 1 & 1 & 1 \\
\hline$u_{2}$ & 1 & 1 & 1 & 2 \\
\hline$u_{3}$ & 1 & 1 & 2 & 3 \\
\hline$u_{4}$ & 1 & 2 & 3 & 4 \\
\hline
\end{tabular}

Figure 3: The pairing $\mathcal{W}(\mathcal{S})$ representing the chain of 4 elements. 


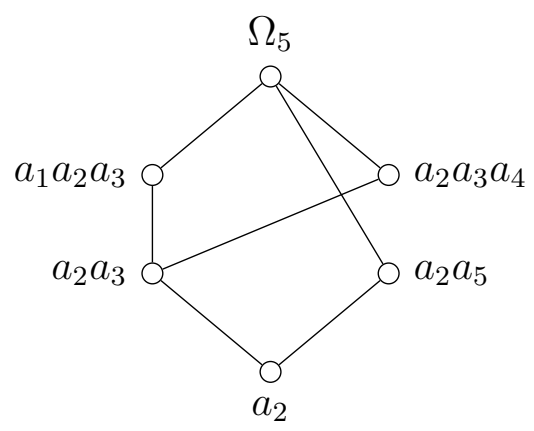

Figure 4: The Hasse Diagram of the closure lattice of Example 7.

The minimum in $\mathbb{S}$ is $E=\left\{a_{2}\right\}$. The lattice associated with $\mathcal{S}$ has the Hasse diagram represented in Figure 4.

There are three maximal chains from $E$ to $\Omega$ :

$$
\begin{aligned}
& \mathcal{C}_{1}: E=A_{1,1}=\left\{a_{2}\right\} \varsubsetneqq A_{1,2}=\left\{a_{2}, a_{3}\right\} \varsubsetneqq A_{1,3}=\left\{a_{1}, a_{2}, a_{3}\right\} \varsubsetneqq A_{1,4}=\Omega_{5} \\
& \mathcal{C}_{2}: E=A_{2,1}=\left\{a_{2}\right\} \varsubsetneqq A_{2,2}=\left\{a_{2}, a_{3}\right\} \varsubsetneqq A_{2,3}=\left\{a_{2}, a_{3}, a_{4}\right\} \varsubsetneqq A_{2,4}=\Omega_{5} \\
& \mathcal{C}_{3}: E=A_{3,1}=\left\{a_{2}\right\} \varsubsetneqq A_{3,2}=\left\{a_{2}, a_{5}\right\} \varsubsetneqq A_{3,3}=\Omega_{5}
\end{aligned}
$$

Thus $m_{0}=0, m_{1}=4, m_{2}=8$ and $m=m_{3}=11$. Moreover $\zeta_{1, a_{1}}=3, \zeta_{2, a_{1}}=4, \zeta_{3, a_{1}}=3$, $\zeta_{1, a_{3}}=2, \zeta_{2, a_{3}}=2, \zeta_{3, a_{3}}=3, \zeta_{1, a_{4}}=4, \zeta_{2, a_{4}}=3, \zeta_{3, a_{4}}=3, \zeta_{1, a_{5}}=4, \zeta_{2, a_{5}}=4$ and $\zeta_{3, a_{5}}=2$. Then

$$
U=\left\{u_{1}:=1, u_{2}:=2, \ldots, u_{11}:=11\right\}
$$

and,

$$
\iota_{1}=\iota_{2}=\iota_{3}=\iota_{4}=1, \iota_{5}=\iota_{6}=\iota_{7}=\iota_{8}=2, \iota_{9}=\iota_{10}=\iota_{11}=3 .
$$

As has already been done in Example 6, in the table represented in Figure 5, we show as acts the algorithm which defines the map $F_{\mathcal{S}}$ given in (10) relatively to the closure system in the present example.

At this point we can construct the pairing represented in Figure 6.

Moreover, in Figure 7 we have also drawn the Hasse diagram of $\mathbb{G}(\mathcal{W}(\mathcal{S})$ ) (we use string notation to represent the subsets of $\Omega_{5}$ ).

Let us provide simple consequences of Theorem 5 .

Corollary 8. Let $\mathcal{S}$ be a closure system on $\Omega$ and $\mathcal{W}$ be a pairing on $\Omega$ such that $\mathcal{M}(\mathcal{W})=$ $\mathcal{S}$. Then the lattices $\mathbb{P}_{\text {sym }}(\mathcal{W}), \mathbb{M}(\mathcal{W})$ and the closure lattice $\mathbb{S}$ are order-isomorphic.

Proof. The result is a direct consequence of Theorem 5 and of part $(v)$ of Theorem 3.

Remark 9. The algorithm provided in Theorem 5, in general, does not yield a pairing with the cardinality of $U_{\mathcal{S}}$ as least as possible. 


\begin{tabular}{|l|l|l|l|l|l|}
\hline & $a_{1}$ & $a_{2}$ & $a_{3}$ & $a_{4}$ & $a_{5}$ \\
\hline$u_{1}$ & $1 \downarrow$ & 1 & $1 \downarrow$ & $1 \downarrow$ & $1 \downarrow$ \\
\hline$u_{2}$ & $u_{2} \in[2,2] \rightarrow 1 \downarrow$ & 1 & $u_{2} \in[2,3] \rightarrow 1 \downarrow$ & $u_{2} \notin \emptyset \rightarrow 1+1 \downarrow$ & $u_{2} \notin \emptyset \rightarrow 1+1 \downarrow$ \\
\hline$u_{3}$ & $u_{3} \notin[2,2] \rightarrow 1+1 \downarrow$ & 1 & $u_{3} \in[2,3] \rightarrow 1 \downarrow$ & $u_{3} \notin \emptyset \rightarrow 2+1 \downarrow$ & $u_{3} \notin \emptyset \rightarrow 2+1 \downarrow$ \\
\hline$u_{4}$ & $u_{4} \notin[2,2] \rightarrow 2+1 \downarrow$ & 1 & $u_{4} \notin[2,3] \rightarrow 1+1 \downarrow$ & $u_{4} \notin \emptyset \rightarrow 3+1 \downarrow$ & $u_{4} \notin \emptyset \rightarrow 3+1 \downarrow$ \\
\hline$u_{5}$ & $u_{5} \notin \emptyset \rightarrow 3+1 \downarrow$ & 1 & $u_{5} \notin[6,7] \rightarrow 2+1 \downarrow$ & $u_{5} \notin[6,6] \rightarrow 4+1 \downarrow$ & $u_{5} \notin \emptyset \rightarrow 4+1 \downarrow$ \\
\hline$u_{6}$ & $u_{6} \notin \emptyset \rightarrow 4+1 \downarrow$ & 1 & $u_{6} \in[6,7] \rightarrow 3 \downarrow$ & $u_{6} \in[6,6] \rightarrow 5 \downarrow$ & $u_{6} \notin \emptyset \rightarrow 5+1 \downarrow$ \\
\hline$u_{7}$ & $u_{7} \notin \emptyset \rightarrow 5+1 \downarrow$ & 1 & $u_{7} \in[6,7] \rightarrow 3 \downarrow$ & $u_{7} \notin[6,6] \rightarrow 5+1 \downarrow$ & $u_{7} \notin \emptyset \rightarrow 6+1 \downarrow$ \\
\hline$u_{8}$ & $u_{8} \notin \emptyset \rightarrow 6+1 \downarrow$ & 1 & $u_{8} \notin[6,7] \rightarrow 3+1 \downarrow$ & $u_{8} \notin[6,6] \rightarrow 6+1 \downarrow$ & $u_{8} \notin \emptyset \rightarrow 7+1 \downarrow$ \\
\hline$u_{9}$ & $u_{9} \notin \emptyset \rightarrow 7+1 \downarrow$ & 1 & $u_{9} \notin \emptyset \rightarrow 4+1 \downarrow$ & $u_{9} \notin \emptyset \rightarrow 7+1 \downarrow$ & $u_{9} \notin[10,10] \rightarrow 8+1 \downarrow$ \\
\hline$u_{10}$ & $u_{10} \notin \emptyset \rightarrow 8+1 \downarrow$ & 1 & $u_{10} \notin \emptyset \rightarrow 5+1 \downarrow$ & $u_{10} \notin \emptyset \rightarrow 8+1 \downarrow$ & $u_{10} \in[10,10] \rightarrow 9 \downarrow$ \\
\hline$u_{11}$ & $u_{11} \notin \emptyset \rightarrow 9+1$ & 1 & $u_{11} \notin[6,7] \rightarrow 6+1$ & $u_{11} \notin \emptyset \rightarrow 9+1$ & $u_{11} \notin[10,10] \rightarrow 9+1$ \\
\hline
\end{tabular}

Figure 5: A visualization of the recursive construction of the map $F_{\mathcal{E}}$ for the closure system of Example 7.

\begin{tabular}{|l|l|l|l|l|l|}
\hline & $a_{1}$ & $a_{2}$ & $a_{3}$ & $a_{4}$ & $a_{5}$ \\
\hline$u_{1}$ & 1 & 1 & 1 & 1 & 1 \\
\hline$u_{2}$ & 1 & 1 & 1 & 2 & 2 \\
\hline$u_{3}$ & 2 & 1 & 1 & 3 & 3 \\
\hline$u_{4}$ & 3 & 1 & 2 & 4 & 4 \\
\hline$u_{5}$ & 4 & 1 & 3 & 5 & 5 \\
\hline$u_{6}$ & 5 & 1 & 3 & 5 & 6 \\
\hline$u_{7}$ & 6 & 1 & 3 & 6 & 7 \\
\hline$u_{8}$ & 7 & 1 & 4 & 7 & 8 \\
\hline$u_{9}$ & 8 & 1 & 5 & 8 & 9 \\
\hline$u_{10}$ & 9 & 1 & 6 & 9 & 9 \\
\hline$u_{11}$ & 10 & 1 & 7 & 10 & 10 \\
\hline
\end{tabular}

Figure 6: The Pairing $\mathcal{W}(\mathcal{S})$ associated with the closure lattice of Example 3.3. 


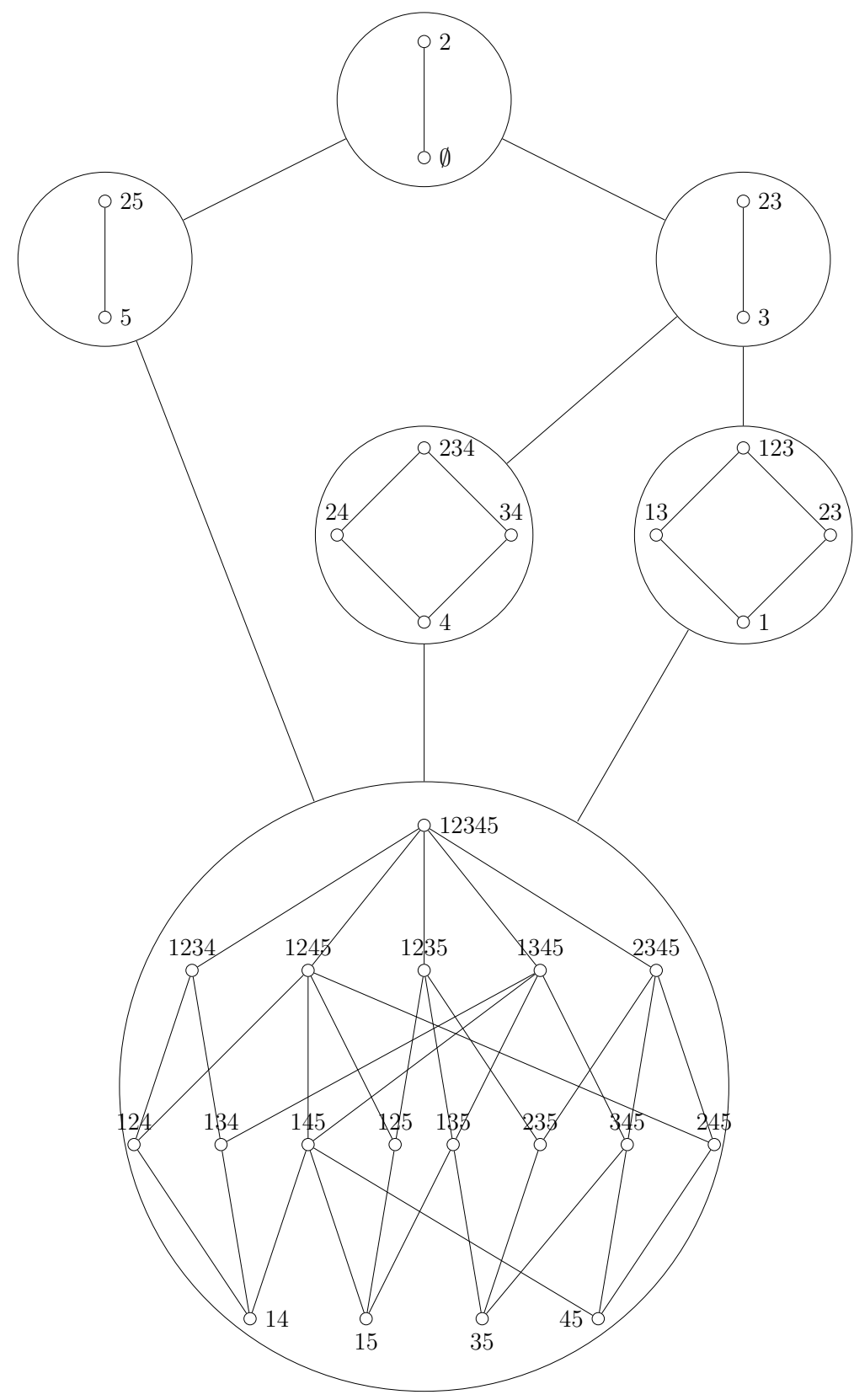

Figure 7: Hasse Diagram of $\mathbb{G}(\mathcal{W}(\mathcal{S})$ ) relative to Example 3.3. 
Remark 10. A very natural question related to the algorithm introduced in Theorem 5 is about its complexity. It is a hard computational problem and it will not be analyzed in this paper. In order to face it, we could divide it into smaller problems and solve each one of them separately. The first of this problem is known as the Moore problem, in honour to E. H. Moore for its work on closure systems (see [27]). It consists of the computation of the number of closure systems on a given finite set $\Omega$ with $n$ elements. Already for $n=7$, this number becomes too large and difficult to be found by an enumeration algorithm. By the way, as proved by Alekseev in [3] its asymptotic size is $O\left(2^{\left(\frac{n}{2}\right)}\right)$. The second part of the problem consists of the computation of all chains in any of the previous closure systems. Therefore, in each case, we have to compute the cost of the implementation of the algorithm and, next, to analyze the average complexity or, at least, an estimation of it.

Relatively to a given pairing $\mathcal{W}$ on $\Omega$, we now consider three set operators

$$
\phi_{\mathcal{W}}, K_{\mathcal{W}}, N_{\mathcal{W}}: \mathcal{P}(\Omega) \longrightarrow \mathcal{P}(\Omega),
$$

defined by:

$$
\phi_{\mathcal{W}}(A):=M_{\mathcal{W}}\left(A^{c}\right), \quad K_{\mathcal{W}}(A):=\left(\phi_{\mathcal{W}}(A)\right)^{c} \text { and } N_{\mathcal{W}}(A):=M_{\mathcal{W}}\left(K_{\mathcal{W}}(A)\right),
$$

and the poset $\mathbb{E}(\mathcal{W}):=(\mathcal{E}(\mathcal{W}), \subseteq)$, where

$$
\mathcal{E}(\mathcal{W}):=\left\{A \in \mathcal{P}(\Omega): A=N_{\mathcal{W}}(A)\right\}=\operatorname{Fix}\left(N_{\mathcal{W}}\right)
$$

The members of the latter set system will be called normally extensible subsets of the pairing $\mathcal{W}$ and will be relevant in the proof of Theorem 22. In the next result, we shall describe some basic properties of the poset $\mathcal{E}(\mathcal{W})$ and of the set operator $K_{\mathcal{W}}$ which we shall use in the last theorem of the paper. In what follows, we say that a pairing $\mathcal{W}$ on $\Omega$ is regular if $M_{\mathcal{W}}(\emptyset)=\emptyset$.

Proposition 11. Let $A, B, C \in \mathcal{P}(\Omega)$ and $\mathcal{W}$ be a pairing on $\Omega$. Then:

(i) $K_{\mathcal{W}}$ is a kernel operator on $\Omega$ and $K_{\mathcal{W}}(A)=\max \{Z \subseteq A: \Omega \backslash Z \in \mathcal{M}(\mathcal{W})\}$;

(ii) if $C$ is the complement of some maximum partitioner, then $M_{\mathcal{W}}(C) \in \mathcal{E}(\mathcal{W})$;

(iii) if $A$ and $A^{c}$ both belong to $\mathcal{M}(\mathcal{W})$ then $A \in \mathcal{E}(\mathcal{W})$;

Assume now that $\mathcal{W}$ is a regular pairing on $\Omega$. Then:

(iv) $\mathbb{E}(\mathcal{W})$ is a complete lattice with $\hat{0}_{\mathbb{E}(\mathcal{W})}=\emptyset$ and $\hat{1}_{\mathbb{E}(\mathcal{W})}=\Omega$;

(v) $\phi_{\mathcal{W}}(\mathcal{E}(\mathcal{W})) \subseteq \mathcal{E}(\mathcal{W})$;

(vi) if $A \subseteq B$, then $\phi_{\mathcal{W}}(A) \geqslant_{\mathcal{W}} \phi_{\mathcal{W}}(B)$;

(vii) $\phi_{\mathcal{W}}$ is an involutory map on $\mathcal{E}(\mathcal{W})$ such that $\phi_{\mathcal{W}}(A) \wedge A=\emptyset$ and $\phi_{\mathcal{W}}(A) \vee A=\Omega$.

Proof. (i): The set operator $K_{\mathcal{W}}$ is clearly intensive and isotone. Furthermore, the idempotence of $K_{\mathcal{W}}$ follows by the equalities:

$$
K_{\mathcal{W}}\left(K_{\mathcal{W}}(A)\right)=\Omega \backslash M_{\mathcal{W}}\left(\Omega \backslash K_{\mathcal{W}}(A)\right)=\Omega \backslash M_{\mathcal{W}}\left(\Omega \backslash\left(\Omega \backslash M_{\mathcal{W}}\left(A^{c}\right)\right)\right)=\Omega \backslash M_{\mathcal{W}}\left(A^{c}\right)=K_{\mathcal{W}}(A) .
$$


To prove the second part of the claim, take $Z \subseteq A$ such that $Z^{c} \in \mathcal{M}(\mathcal{W})$ and assume that $Z=\Omega \backslash M_{\mathcal{W}}(B)$ for some $B \in \mathcal{P}(\Omega)$. We claim that $Z \subseteq K_{\mathcal{W}}(A)$. In fact, the condition $Z=\Omega \backslash M_{\mathcal{W}}(B) \subseteq A$ implies that $M_{\mathcal{W}}(B) \supseteq A^{c}$, i.e. $M_{\mathcal{W}}(B) \supseteq M_{\mathcal{W}}\left(A^{c}\right)$, so $Z \subseteq \Omega \backslash M_{\mathcal{W}}\left(A^{c}\right)=K_{\mathcal{W}}(A)$

(ii): Let $C=\Omega \backslash M_{\mathcal{W}}(B)$ for some $B \in \mathcal{P}(\Omega)$. By the above part $(i)$, we get $C \subseteq$ $K_{\mathcal{W}}\left(M_{\mathcal{W}}(C)\right) \subseteq M_{\mathcal{W}}(C)$, whence

$$
N_{\mathcal{W}}\left(M_{\mathcal{W}}(C)\right)=M_{\mathcal{W}}\left(K_{\mathcal{W}}\left(M_{\mathcal{W}}(C)\right)\right)=M_{\mathcal{W}}(C),
$$

i.e. $M_{\mathcal{W}}(C) \in \mathcal{E}(\mathcal{W})$.

(iii): As $A, A^{c} \in \mathcal{M}(\mathcal{W})$, we get $K_{\mathcal{W}}(A)=\left(M_{\mathcal{W}}\left(A^{c}\right)\right)^{c}=A$, whence $N_{\mathcal{W}}(A)=A$, i.e. $A \in \mathcal{E}(\mathcal{W})$.

(iv): As $\mathcal{W}$ is regular, then $\emptyset, \Omega \in \mathcal{E}(\mathcal{W})$. Let now $\mathcal{L} \subseteq \mathcal{E}(\mathcal{W})$. Using the above part (ii) and the properties of $K_{\mathcal{W}}$ and $M_{\mathcal{W}}$, it may be easily shown that

$$
\bigwedge \mathcal{L}=M_{\mathcal{W}}\left(K_{\mathcal{W}}(\bigcap \mathcal{L})\right)=N_{\mathcal{W}}(\bigcap \mathcal{L}) \text { and } \bigvee \mathcal{L}=M_{\mathcal{W}}\left(\bigcup\left\{K_{\mathcal{W}}(B): B \in \mathcal{L}\right\}\right)
$$

$(v)$ : Let $A \in \mathcal{E}(\mathcal{W})$. Since $A \in \mathcal{M}(\mathcal{W})$, then, by part $(i i)$, we get $M_{\mathcal{W}}\left(A^{c}\right) \in \mathcal{E}(\mathcal{W})$.

(vi): The map $\phi_{\mathcal{W}}$ is order-reversing, in fact if $A \subseteq B$, then $\Omega \backslash B \subseteq \Omega \backslash A$, i.e. $M_{\mathcal{W}}(\Omega \backslash B) \subseteq$ $M_{\mathcal{W}}(\Omega \backslash A)$ or, in terms of union additive relations, $M_{\mathcal{W}}(\Omega \backslash A) \geqslant{ }_{\mathcal{W}} M_{\mathcal{W}}(\Omega \backslash B)$, i.e. $\phi_{\mathcal{W}}(A) \geqslant_{\mathcal{W}} \phi_{\mathcal{W}}(B)$.

(vii): Let $A \in \mathcal{E}(\mathcal{W})$. The fact that $\phi_{\mathcal{W}}$ is an involutory map follows by the equalities $\phi_{\mathcal{W}}\left(\phi_{\mathcal{W}}(A)\right)=\phi_{\mathcal{W}}\left(M_{\mathcal{W}}\left(A^{c}\right)\right)=M_{\mathcal{W}}\left(\Omega \backslash M_{\mathcal{W}}\left(A^{c}\right)\right)=N_{\mathcal{W}}(A)=A$.

Let us prove that $\phi_{\mathcal{W}}(A) \wedge A=\emptyset$. Using the first relation in (23), we get

$$
\phi_{\mathcal{W}}(A) \wedge A=N_{\mathcal{W}}\left(\phi_{\mathcal{W}}(A) \cap A\right)=N_{\mathcal{W}}\left(M_{\mathcal{W}}\left(A^{c}\right) \cap A\right) .
$$

We now prove that the empty set is the only subset contained in $M_{\mathcal{W}}\left(A^{c}\right) \cap A$ and whose complement is a maximum partitioner. As a matter of fact, let $Z \subseteq M_{\mathcal{W}}\left(A^{c}\right) \cap A$, then $Z \subseteq A$ and, in particular, in view of part $(i)$, we get $Z \subseteq K_{\mathcal{W}}(A)=\Omega \backslash M_{\mathcal{W}}\left(A^{c}\right)$. But it happens if and only if $Z=\emptyset$. Thus, $K_{\mathcal{W}}\left(M_{\mathcal{W}}\left(A^{c}\right) \cap A\right)=\emptyset$ and, since $M_{\mathcal{W}}(\emptyset)=\emptyset$, we conclude that $\phi_{\mathcal{W}}(A) \wedge A=M_{\mathcal{W}}(\emptyset)=\emptyset$.

On the other hand, let us compute $\phi_{\mathcal{W}}(A) \vee A$. Using the second relation in (23), we get

$$
\begin{gathered}
\phi_{\mathcal{W}}(A) \vee A=M_{\mathcal{W}}\left(K_{\mathcal{W}}\left(\phi_{\mathcal{W}}(A)\right) \cup K_{\mathcal{W}}(A)\right) \supseteq \\
\supseteq M_{\mathcal{W}}\left(K_{\mathcal{W}}\left(\phi_{\mathcal{W}}(A)\right)\right) \cup M_{\mathcal{W}}\left(K_{\mathcal{W}}(A)\right)=N_{\mathcal{W}}\left(\phi_{\mathcal{W}}(A)\right) \cup N_{\mathcal{W}}(A) .
\end{gathered}
$$

Now, as $A, \phi_{\mathcal{W}}(A) \in \mathcal{E}(\mathcal{W})$, we conclude that

$$
\phi_{\mathcal{W}}(A) \vee A \supseteq \phi_{\mathcal{W}}(A) \cup A \supseteq \Omega \text {. }
$$

This shows that $\phi_{\mathcal{W}}(A) \vee A=\Omega$ and the proof concludes here.

We close this section with another consequence of Theorem 5 . We shall see that given a complement-closed set system on $\Omega$, we can find a regular pairing whose maximum partitioners agree with the fixed points of $I n t_{\mathcal{F}}$. 
Proposition 12. Let $\mathcal{F}$ be a complement-closed set system on $\Omega$. Then there exists a regular pairing $\mathcal{W}$ on $\Omega$ such that:

- $\mathcal{M}(\mathcal{W})=F i x\left(\right.$ Int $\left._{\mathcal{F}}\right)$;

- $\mathcal{F} \subseteq \mathcal{M}(\mathcal{W})$;

- $K_{\mathcal{W}}(A)=A$ for each $A \in \mathcal{F}$.

Proof. Let $\mathcal{F}$ be a complement-closed set system on $\Omega$. In view of Proposition 2, we have that the set operator $\operatorname{Int}_{\mathcal{F}}$ is a closure operator on $\Omega$ and, hence, Fix $\left(\operatorname{Int}_{\mathcal{F}}\right)$ is a closure system on $\Omega$ by Theorem 1 . Now, Theorem 5 ensures the existence of a pairing $\mathcal{W}$ on $\Omega$ such that $\mathcal{M}(\mathcal{W})$ agrees with the family of all the fixed subsets of $\operatorname{Int}_{\mathcal{F}}$, i.e. $\mathcal{M}(\mathcal{W})=$ Fix $\left(\right.$ Int $\left._{\mathcal{F}}\right)$.

At this point, take $A \in \mathcal{F}$. In view of the definition of $\operatorname{Int}_{\mathcal{F}}$, it results that $A=\operatorname{Int}_{\mathcal{F}}(A)=$ $M_{\mathcal{W}}(A)$, i.e. $\mathcal{F} \subseteq \mathcal{M}(\mathcal{W})$. In particular, $\emptyset=\operatorname{Int}_{\mathcal{F}}(\emptyset)=M_{\mathcal{W}}(\emptyset)$, whence the regularity of the pairing $\mathcal{W}$.

Finally, we must show that $K_{\mathcal{W}}(A)=A$. To this regard, notice first that $A^{c} \in \mathcal{F}$ since $\mathcal{F}$ is a complement-closed family. Thus, $M_{\mathcal{W}}\left(A^{c}\right)=\operatorname{Int}_{\mathcal{F}}\left(A^{c}\right)=A^{c}$ and $\left(A^{c}\right)^{c}=A$. Therefore $K_{\mathcal{W}}(A)=\left(M_{\mathcal{W}}\left(A^{c}\right)\right)^{c}=A$, as wanted.

\section{Union Additive Relations and a Related Lattice Representa- tion Theorem}

In this section we will introduce the notion of a union additive relation on an arbitrary set $\Omega$, namely a preorder relation $\geqslant$ on $\mathcal{P}(\Omega)$ satisfying property (4). We will see that a pairing induces a union additive relation and that, in general, any union additive relation on $\Omega$ agrees with the preorder $\geqslant_{\mathcal{W}}$ induced by some pairing $\mathcal{W}$ on $\Omega$. We will obtain such a result as a consequence of Theorem 5. Another consequence of the aforementioned theorem consists of the possibility of representing any finite lattice $\mathbb{L}$ as the maximum partitioner lattice of some pairing $\mathcal{W}_{\mathbb{L}}$ on a specific finite ground set $\Omega_{\mathbb{L}}$.

First of all, we introduce the following basic notion.

Definition 13. We call a binary relation $\geqslant$ on $\mathcal{P}(\Omega)$ such that:

(D1) if $X \supseteq Y$, then $X \geqslant Y$;

(D2) if $X \geqslant Y_{i} \forall i \in I$, then $X \geqslant \bigcup Y_{i}$;

(D3) if $X \geqslant Y$ and $Y \geqslant Z$, then $X \geqslant Z$.

a union additive relation on $\Omega$.

Theorem 3 enables us to find a union additive relation in the context of pairings. As a matter of fact, we can set:

$$
A \geqslant_{\mathcal{W}} A^{\prime}: \Longleftrightarrow M_{\mathcal{W}}(A) \supseteq M_{\mathcal{W}}\left(A^{\prime}\right) .
$$


and it may be easily verified that $\geqslant_{\mathcal{W}}$ is a union additive relation on $\Omega$, which we call the $\mathcal{W}$-union additive relation on $\Omega$. Moreover, we may interpret the relation $\approx_{\mathcal{W}}$ as the equivalence relation induced by the preorder $\geqslant_{w}$, i.e.

$$
A \approx_{\mathcal{W}} A^{\prime} \Longleftrightarrow A \geqslant_{\mathcal{W}} A^{\prime} \text { and } A^{\prime} \geqslant_{\mathfrak{W}} A
$$

Consequently, if we consider the preordered set $\mathbb{H}(\mathcal{W}):=(\mathcal{P}(\Omega), \geqslant \mathcal{W})$, we immediately obtain the following order isomorphisms.

Proposition 14. We have that $\mathbb{H}(\mathcal{W}) / \approx_{\mathcal{W}} \cong \mathbb{M}(\mathcal{W}) \cong \mathbb{P}_{\text {sym }}(\mathcal{W})$.

A consequence of Theorem 5 consists of the possibility to represent any union additive relation on $\Omega$ as the preorder relation $\geqslant_{\mathcal{W}}$ induced by some pairing on $\Omega$ itself.

Theorem 15. Let $\geqslant$ be a union additive relation on $\Omega$. Then there exists a pairing $\mathcal{W}$ on $\Omega$ such that $\geqslant$ coincides with $\geqslant$ w.

Proof. Let $\geqslant$ be a given union additive relation on $\Omega$. Consider the set operator $\sigma \geqslant$ defined as follows:

$$
\sigma_{\geqslant}(A):=\bigcup\{B \in \mathcal{P}(\Omega): A \geqslant B\}
$$

for each $A \in \mathcal{P}(\Omega)$. It may be easily verified that $\sigma_{\geqslant}$is a closure operator on $\Omega$. At this point, Theorem 1 ensures that the set system of fixed points of $\sigma \geqslant$ forms a closure system $\mathcal{S}$ on $\Omega$. Therefore, in view of Theorem 5 , there exists a pairing $\mathcal{W}$ on $\Omega$ such that $\mathcal{M}(\mathcal{W})=\mathcal{S}$ and, in particular, by Theorem 1 , we also infer that $M_{\mathcal{W}}$ agrees with $\sigma_{\geqslant}$. Finally, since $A \geqslant_{\mathcal{W}} B$ if and only if $M_{\mathcal{W}}(B) \subseteq M_{\mathcal{W}}(A)=\sigma_{\geqslant}(A)$, we must necessarily have $A \geqslant B$ if and only if $A \geqslant w B$.

By means of Theorem 5, in what follows we will be able to show that any finite lattice is order-isomorphic to the maximum partitioner lattice of some pairing on a specific ground set, and, moreover, that any union additive relation on $\Omega$ can be represented by the preorder $\geqslant_{\mathcal{W}}$ induced by some pairing $\mathcal{W}$ on $\Omega$. To this regard, we firstly recall some classical notions of database theory that are used to understand the degree of dependency of two attribute subsets of a relational database (see [34] for details).

If $A, B \in \mathcal{P}(\Omega)$, we set

$$
\Gamma_{\mathcal{W}}(A, B):=\left\{u \in U:[u]_{A} \subseteq[u]_{B}\right\},
$$

and, if $U$ is finite,

$$
\gamma_{\mathcal{W}}(A, B):=\frac{\left|\Gamma_{\mathcal{W}}(A, B)\right|}{|U|}
$$

Some basic properties of the set operator $\Gamma_{\mathcal{W}}$ are described in the next proposition, whose proof is straightforward. 
Proposition 16. Let $A, A^{\prime}, B, B^{\prime} \in \mathcal{P}(\Omega)$. Then the following hold:

(i) $A \geqslant_{\mathcal{W}} A^{\prime} \Longleftrightarrow \pi_{\mathcal{W}}(A) \preceq \pi_{\mathcal{W}}\left(A^{\prime}\right) \Longleftrightarrow \Gamma_{\mathcal{W}}\left(A, A^{\prime}\right)=U \Longleftrightarrow \gamma_{\mathcal{W}}\left(A, A^{\prime}\right)=1$;

(ii) If $A \subseteq A^{\prime}$ and $B \subseteq B^{\prime}$, then

$$
\Gamma_{\mathcal{W}}(A, B) \subseteq \Gamma_{\mathcal{W}}\left(A^{\prime}, B\right) \text { and } \Gamma_{\mathcal{W}}(A, B) \supseteq \Gamma_{\mathcal{W}}\left(A, B^{\prime}\right)
$$

(iii) $M_{\mathcal{W}}(A)=\left\{b \in \Omega: \Gamma_{\mathcal{W}}(A,\{b\})=U\right\}$.

At this point, based on a classical representation theorem of lattice theory (see [6]), we can establish the following pairing representation theorem for finite lattices.

Theorem 17. Let $\mathbb{L}=\left(L, \leqslant_{\mathbb{L}}\right)$ be a finite lattice. Then there exist a finite set $\Omega_{\mathbb{L}}$ and a pairing $\mathcal{W}$ on $\Omega_{\mathbb{L}}$ such that the lattices $\mathbb{L}, \mathbb{H}(\mathcal{W}) / \approx_{\mathcal{W}}$ and $\mathbb{M}(\mathcal{W})$ are order-isomorphic. Therefore, if $x, x^{\prime} \in L$ and $\eta_{\mathcal{W}}: L \rightarrow \mathcal{M}(\mathcal{W})$ is the map that induces such an isomorphism between $\mathbb{L}$ and $\mathbb{M}(\mathcal{W})$, the following conditions are equivalent:

- $x \leqslant \mathbb{L} x^{\prime}$

- $\eta_{\mathcal{W}}(x) \subseteq^{*} \eta_{\mathcal{W}}\left(x^{\prime}\right)$

- $\pi_{\mathcal{W}}\left(\eta_{\mathcal{W}}(x)\right) \preceq \pi_{\mathcal{W}}\left(\eta_{\mathcal{W}}\left(x^{\prime}\right)\right)$;

- $\gamma_{\mathcal{W}}\left(\eta_{\mathcal{W}}(x), \eta_{\mathcal{W}}\left(x^{\prime}\right)\right)=1$.

Proof. Before tackling the proof, let us recall that any finite lattice $\mathbb{L}$ is order-isomorphic to a closure lattice induced by a specific closure system on the set $\Omega_{\mathbb{L}}:=\mathcal{J}(\mathbb{L})$, where $\mathcal{J}(\mathbb{L})$ is the set of all join-irreducible elements of $\mathbb{L}$ (see [6]). Such a closure system consists of the set system of the fixed points of the closure operator $\phi: \mathcal{P}\left(\Omega_{\mathbb{L}}\right) \rightarrow \mathcal{P}\left(\Omega_{\mathbb{L}}\right)$ defined as follows:

$$
\phi(A):=\left\{x \in \Omega_{\mathbb{L}}: x \leqslant \bigvee A\right\} .
$$

Therefore, we set $\Omega_{\mathbb{L}}:=\mathcal{J}(\mathbb{L})$. We also denote by $\mathcal{S}_{\mathbb{L}}$ the resulting closure system and by $\eta$ : $L \rightarrow \mathcal{S}_{\mathbb{L}}$ the order isomorphism between the dual lattice $\mathbb{L}^{*}$ and the lattice $\left(\mathcal{S}_{\mathbb{L}}, \subseteq\right)$. Then, by Theorem 5 , we can construct a pairing $\mathcal{W}$ on $\Omega_{\mathbb{L}}$ such that $\mathcal{M}(\mathcal{W})=\mathcal{S}_{\mathbb{L}}$. Therefore $\eta_{\mathcal{W}}:=\eta$ is an order isomorphism between $\mathbb{L}$ and $\mathbb{M}(\mathcal{W})$, and the wanted equivalences become a direct consequence of part $(i)$ of Proposition 16.

The equivalences established in Theorem 17 has some theoretical consequences: it says us that the study of union additive relations between subsets of finite sets is equivalent to the study of order relations on finite lattices. Therefore in the next part of this work we try to investigate the basic theoretical properties of the notion of union additive relations between subsets of finite sets and the direct interrelation of this notion with other classical notions of lattice theory.

We call pairing characteristic of the lattice $\mathbb{L}$, denoted by $p c(\mathbb{L})$, the minimum allowable cardinality of the set $\Omega_{\mathbb{L}}$ obtained from the thesis of Theorem 17 .

Let $N:=p c(\mathbb{L})$. We denote by $\mathcal{P} \mathcal{L}(\mathbb{L})$ the collection of all pairings on $\Omega_{N}$ such that $\mathbb{M}(\mathcal{W}) \cong \mathbb{L}$. Now, if $\mathcal{W} \in \mathcal{P} \mathcal{L}(\mathbb{L})$, with the same notations introduced in the statement 
of Theorem 17, we can consider the inverse order isomorphism $\eta_{\mathcal{W}}^{-1}: \mathcal{M}(\mathcal{W}) \rightarrow L$ and the closure map $M_{\mathcal{W}}: \mathcal{P}\left(\Omega_{N}\right) \rightarrow \mathcal{M}(\mathcal{W})$, so that we obtain the surjective map

$$
\xi_{\mathcal{W}}:=\eta_{\mathcal{W}}^{-1} \circ M_{\mathcal{W}}: \mathcal{P}\left(\Omega_{N}\right) \rightarrow L .
$$

Let us note that the map $\xi_{\mathcal{W}}$ is not canonically determined. In fact, it depends from the order isomorphism $\eta_{\mathcal{W}}$, which in turn depends on the not uniquely determined closure system $\mathcal{S}$ given in the proof of Theorem 17 . However, by means of the map $\xi_{\mathcal{w}}$ we can formally describe the following equivalences.

Theorem 18. Let $\mathbb{L}=\left(L, \leqslant_{\mathbb{L}}\right)$ be a finite lattice, $N=p c(\mathbb{L})$ and $X, Y \in \mathcal{P}\left(\Omega_{N}\right)$. Then the following conditions are equivalent:

(i) $\xi_{\mathcal{W}}(X) \leqslant_{\mathbb{L}} \xi_{\mathcal{W}}(Y)$;

(ii) for any $\mathcal{W} \in \mathcal{P} \mathcal{L}(\mathbb{L})$ we have $\gamma_{\mathcal{W}}(X, Y)=1$;

(iii) there exists $\mathcal{W} \in \mathcal{P} \mathcal{L}(\mathbb{L})$ such that $\gamma_{\mathcal{W}}(X, Y)=1$.

Proof. $(i) \Longrightarrow(i i)$ : Let $x:=\xi_{\mathcal{W}}(X) \leqslant_{\mathbb{L}} y:=\xi_{\mathcal{W}}(Y)$. Let $\mathcal{W}^{\prime} \in \mathcal{P} \mathcal{L}(\mathbb{L})$ and $\eta_{\mathcal{W}^{\prime}}: L \rightarrow$ $\mathcal{M}\left(\mathcal{W}^{\prime}\right)$. In view of the equivalences given in Theorem 17 , we get $\eta_{\mathcal{W}^{\prime}}(x) \subseteq \subseteq^{*} \eta_{\mathcal{W}^{\prime}}(y)$, i.e.

$$
\pi_{\mathcal{W}^{\prime}}\left(\eta_{\mathcal{W}^{\prime}}(x)\right) \preceq \pi_{\mathcal{W}^{\prime}}\left(\eta_{\mathcal{W}^{\prime}}\left(x^{\prime}\right)\right) \Longleftrightarrow \gamma_{\mathcal{W}^{\prime}}\left(\eta_{\mathcal{W}^{\prime}}(x), \eta_{\mathcal{W}^{\prime}}\left(x^{\prime}\right)\right)=1
$$

and the claim has been shown.

$($ ii $) \Longrightarrow($ iii $)$ : Obvious.

$($ iii $) \Longrightarrow(i)$ : In view of the equivalences given in Theorem 17 we have that $\gamma_{\mathcal{W}}(X, Y)=$ $\gamma_{\mathcal{W}}\left(M_{\mathcal{W}}(X), M_{\mathcal{W}}(Y)\right)=1$ if and only if $\pi_{\mathcal{W}}(X) \preceq \pi_{\mathcal{W}}(Y)$ or, equivalently, $M_{\mathcal{W}}(Y) \subseteq$ $M_{\mathcal{W}}(X)$, that is equivalent to $\eta_{\mathcal{W}}^{-1}\left(M_{\mathcal{W}}(X)\right) \preceq \eta_{\mathcal{W}}^{-1}\left(M_{\mathcal{W}}(Y)\right)$, i.e. $\xi_{\mathcal{W}}(X) \leqslant_{\mathbb{L}} \xi_{\mathcal{W}}(Y)$.

Example 19. Let us consider the lattice $M_{3}$ (whose definition has been provided in Section 2, page 4) and let $a_{1}, a_{2}, a_{3}$ be the elements of the lattice that are different from $\hat{0}$ and $\hat{1}$. Then, $M_{3}$ is order-isomorphic to the lattice $\mathbb{S}=(\mathcal{S}, \subseteq)$ induced by the closure system $\mathcal{S}$ on $\Omega_{3}:=\left\{a_{1}, a_{2}, a_{3}\right\}$, where $\mathcal{S}:=\left\{\emptyset,\left\{a_{1}\right\},\left\{a_{2}\right\},\left\{a_{3}\right\}, \Omega_{3}\right\}$. By means of Theorem 5 , we can represent the aforementioned closure system $\mathcal{S}$ through a pairing, that we denote by $\mathcal{W}\left(M_{3}\right)$. In order to build such a pairing and using the notations of Theorem 5 , we get $m_{0}=0, m_{1}=3, m_{2}=6$ and $m_{3}=9$. Set $U:=\left\{u_{1}:=1, \ldots, u_{9}:=9\right\}$.

Moreover, we also obtain $\zeta_{1, a_{1}}=2, \zeta_{2, a_{1}}=3, \zeta_{3, a_{1}}=3, \zeta_{1, a_{2}}=3, \zeta_{2, a_{2}}=2, \zeta_{3, a_{2}}=3$, $\zeta_{1, a_{3}}=3, \zeta_{2, a_{3}}=3$ and $\zeta_{3, a_{3}}=2$. So, we represent the pairing $\mathcal{W}\left(M_{3}\right)$ as a table in Figure 8.

A quick computation of the symmetry partitions gives the following result:

$$
\begin{gathered}
\pi_{\mathcal{W}}(\emptyset)=U ; \quad \pi_{\mathcal{W}}\left(\left\{a_{1}\right\}\right)=u_{1} u_{2}\left|u_{3}\right| u_{4}\left|u_{5}\right| u_{6}\left|u_{7}\right| u_{8} \mid u_{9} ; \\
\pi_{\mathcal{W}}\left(\left\{a_{2}\right\}\right)=u_{1}\left|u_{2}\right| u_{3}\left|u_{4} u_{5}\right| u_{6}\left|u_{7}\right| u_{8}\left|u_{9} ; \quad \pi_{\mathcal{W}}\left(\left\{a_{3}\right\}\right)=u_{1}\right| u_{2}\left|u_{3}\right| u_{4}\left|u_{5}\right| u_{6}\left|u_{7} u_{8}\right| u_{9} ; \\
\pi_{\mathcal{W}}\left(\Omega_{3}\right)=u_{1}\left|u_{2}\right| u_{3}\left|u_{4}\right| u_{5}\left|u_{6}\right| u_{7}\left|u_{8}\right| u_{9} .
\end{gathered}
$$

Moreover, let us provide the table whose entries are the values of $\gamma_{\mathcal{W}}(X, Y)$ for both $X, Y$ varying over $\mathcal{P}\left(\Omega_{3}\right)$. 


\begin{tabular}{|l|l|l|l|}
\hline & $a_{1}$ & $a_{2}$ & $a_{3}$ \\
\hline$u_{1}$ & 1 & 1 & 1 \\
\hline$u_{2}$ & 1 & 2 & 2 \\
\hline$u_{3}$ & 2 & 3 & 3 \\
\hline$u_{4}$ & 3 & 4 & 4 \\
\hline$u_{5}$ & 4 & 4 & 5 \\
\hline$u_{6}$ & 5 & 5 & 6 \\
\hline$u_{7}$ & 6 & 6 & 7 \\
\hline$u_{8}$ & 7 & 7 & 7 \\
\hline$u_{9}$ & 8 & 8 & 8 \\
\hline
\end{tabular}

Figure 8: The pairing $\mathcal{W}\left(M_{3}\right)$.

\begin{tabular}{|l|l|l|l|l|l|l|l|l|}
\hline & $\emptyset$ & $\left\{a_{1}\right\}$ & $\left\{a_{2}\right\}$ & $\left\{a_{3}\right\}$ & $\left\{a_{1}, a_{2}\right\}$ & $\left\{a_{1}, a_{3}\right\}$ & $\left\{a_{2}, a_{3}\right\}$ & $\Omega_{3}$ \\
\hline$\emptyset$ & 1 & 0 & 0 & 0 & 0 & 0 & 0 & 0 \\
\hline$\left\{a_{1}\right\}$ & 1 & 1 & $7 / 9$ & $7 / 9$ & $7 / 9$ & $7 / 9$ & $7 / 9$ & $7 / 9$ \\
\hline$\left\{a_{2}\right\}$ & 1 & $7 / 9$ & 1 & $7 / 9$ & $7 / 9$ & $7 / 9$ & $7 / 9$ & $7 / 9$ \\
\hline$\left\{a_{3}\right\}$ & 1 & $7 / 9$ & $7 / 9$ & 1 & $7 / 9$ & $7 / 9$ & $7 / 9$ & $7 / 9$ \\
\hline$\left\{a_{1}, a_{2}\right\}$ & 1 & 1 & 1 & 1 & 1 & 1 & 1 & 1 \\
\hline$\left\{a_{1}, a_{3}\right\}$ & 1 & 1 & 1 & 1 & 1 & 1 & 1 & 1 \\
\hline$\left\{a_{2}, a_{3}\right\}$ & 1 & 1 & 1 & 1 & 1 & 1 & 1 & 1 \\
\hline$\Omega_{3}$ & 1 & 1 & 1 & 1 & 1 & 1 & 1 & 1 \\
\hline
\end{tabular}

Remark 20. Let us consider the following equivalence relation on the family of all pairings on $\Omega$ : two pairings $\mathcal{W}$ and $\mathcal{W}^{\prime}$ are equivalent whenever their symmetry partition lattices are order-isomorphic. Hence, Theorem 17 ensures that any finite lattice is the symmetry partition lattice of a pairing. In particular, any finite lattice identifies an equivalence class of pairings. Let us note that we have a constructive way in order to associate with a finite lattice a pairing on a suitable set $\Omega$. At this point, by means of Theorem 5 , we represent (again in a constructive way) the closure system as a pairing on $\Omega$. However, for pairings, we have defined the set $\Gamma_{\mathcal{W}}(A, B)$ and its numerical counterpart $\gamma_{\mathcal{W}}(A, B)$ in the finite case. Hence, through the equivalences given in Theorem 17, it is possible to see that, whenever we take a finite lattice $\mathbb{L}$ with $N=p c(\mathbb{L})$ and $\mathcal{W} \in \mathcal{P} \mathcal{L}(\mathbb{L})$, then $\gamma_{\mathcal{W}}$ refines the partial order $\leqslant_{\mathbb{L}}$ of the lattice. In fact, let $x, y \in \mathbb{L}$ be two elements of the lattice. Then it results that $x \leqslant_{\mathbb{L}} y$ if and only if $\gamma_{\mathcal{W}}\left(\eta_{\mathcal{W}}(x), \eta_{\mathcal{W}}(y)\right)=1$, hence we can always compute $\gamma_{\mathcal{W}}\left(\eta_{\mathcal{W}}(x), \eta_{\mathcal{W}}(y)\right)$ even if $x$ and $y$ are non-comparable. In other terms, the function $\gamma_{\mathcal{W}}$ provides extra numerical informations for partial dependencies of subsets, corresponding to two non-comparable nodes of the lattice $\mathbb{L}$ and that are not explicit in the lattice itself. Note that each node corresponds to an equivalence class with respect to the equivalence relation $\approx_{\mathcal{W}}$ and the value of $\gamma_{\mathcal{W}}$ corresponding to a pair of subsets belonging to the same equivalence class is clearly 1.

In general, given a pairing $\mathcal{W}$ on a finite set $\Omega$ with $n$ elements, it is always possible to put within a $2^{n} \times 2^{n}$ table $T(\mathcal{W})$ the values $\gamma_{\mathcal{W}}(X, Y)$. This table is a source of useful 
informations about the properties of the pairing. Indeed, the complete knowledge of all the entries of the table allows us to reconstruct the closure system $\mathcal{M}(\mathcal{W})$, so $T(\mathcal{W})$ represents a numerical completion of $\mathcal{M}(\mathcal{W})$.

\section{Pairing Representation of Finite Complemented Involutory Lattices}

In this section, we prove a second representation theorem for particular types of lattices. To be more detailed, we will consider finite lattices endowed with an order-reversing involutory map $\psi: L \rightarrow L$ exchanging $\hat{0}_{\mathbb{L}}$ and $\hat{1}_{\mathbb{L}}$ and such that $\psi(\alpha) \wedge \alpha=\hat{0}_{\mathbb{L}}$ and $\psi(\alpha) \vee \alpha=\hat{1}_{\mathbb{L}}$ and, next, we show that they are represented by pairings $\mathcal{W}$ on some finite ground set $\Omega_{\mathbb{L}, \psi}$ so that the poset of the normally extensible subsets of $\mathcal{W}$ is orderisomorphic to the starting lattice $\mathbb{L}$.

In Theorem 21 we shall see that starting from a finite complemented involutory lattice, a finite set $\Omega_{n}$, a complement-closed family on $\Omega_{n}$ and an order-preserving $\psi$-complementary map $f: \mathbb{L} \rightarrow(\mathcal{F}, \subseteq)$, we may construct a new finite set, a complement-closed family on it and an order-preserving $\psi$-complementary map from $\mathbb{L}$ to such a family with some specific properties of preservation of pairs of elements of the lattice.

Theorem 21. Let $(\mathbb{L}, \psi)$ be a finite complemented involutory lattice, $\Omega_{n}=\left\{x_{1}, \ldots, x_{n}\right\}$ a fixed finite set, $\mathcal{F}$ a complement-closed family on $\Omega_{n}$ and $f: \mathbb{L} \rightarrow(\mathcal{F}, \subseteq)$ an orderpreserving $\psi$-complementary map. Then, for any $\alpha, \beta \in L$, there exist:

- a finite set $\Omega$,

- a complement-closed family $\mathcal{G}$ on $\Omega$,

- a map $g: \mathbb{L} \rightarrow(\mathcal{G}, \subseteq)$ which is order-preserving, $\psi$-complementary and $\{\alpha, \beta\}$ preserving,

such that:

(A) if $\lambda, \mu \in L$ and $f$ is $\{\lambda, \mu\}$-preserving, then $g$ is also $\{\lambda, \mu\}$-preserving;

(B) $\mathcal{G}=\operatorname{Im}(g)$.

Proof. Let us firstly assume that $f$ is $\{\alpha, \beta\}$-preserving. In such a case, we take $\Omega=\Omega_{n}$ and $g=f$. Moreover, since the image of a finite complemented involutory lattice by an order-preserving $\psi$-complementary map is a complement-closed family, we can also take $\mathcal{G}=\operatorname{Im}(g)$. Thus, the claim holds when $f$ is $\{\alpha, \beta\}$-preserving.

Therefore, we may assume that $f$ is not $\{\alpha, \beta\}$-preserving. Then, five possible cases may occur:

$(C 1) \alpha<_{\mathbb{L}} \beta$ and $f(\alpha)=f(\beta)$;

$(C 2) \beta<_{\mathbb{L}} \alpha$ and $f(\alpha)=f(\beta)$;

$(C 3) \alpha \| \beta$ and $f(\alpha)=f(\beta)$; 
(C4) $\alpha \| \beta$ and $f(\alpha) \varsubsetneqq f(\beta)$;

$(C 5) \alpha \| \beta$ and $f(\beta) \varsubsetneqq f(\alpha)$.

We now examine separately the aforementioned cases.

$(C 1)$ : Let us first extend the set $\Omega_{n}$ by taking a new formal element $x_{n+1} \notin \Omega_{n}$, and we set

$$
\Omega_{n+1}:=\Omega_{n} \cup\left\{x_{n+1}\right\}, h_{0}:=f, \beta_{0}:=\beta
$$

Then we define a map $h_{1}: L \rightarrow \mathcal{P}\left(\Omega_{n+1}\right)$ as follows:

$$
h_{1}(\gamma):= \begin{cases}h_{0}(\gamma) \cup\left\{x_{n+1}\right\} & \text { if } \beta \leqslant \mathbb{L} \gamma \\ h_{0}(\gamma) & \text { otherwise }\end{cases}
$$

for any $\gamma \in L$.

First of all, we observe that $h_{1}$ is an order-preserving map since the starting map $h_{0}$ is order-preserving. Moreover, we get $h_{1}(\alpha) \varsubsetneqq h_{1}\left(\beta_{0}\right)$.

Let us prove now that, whenever $h_{0}$ is $\{\lambda, \mu\}$-preserving for some $\lambda, \mu \in L$, then also $h_{1}$ satisfies the same property. In other terms, we must prove that the restriction of $h_{1}$ to $\{\lambda, \mu\}$ is an order-isomorphism. First of all, let us note that the condition $h_{1}(\lambda)=h_{1}(\mu)$ implies $f(\lambda)=f(\mu)$, whence $\lambda=\mu$. At this point, we shall show that the condition $\lambda \| \mu$ implies $h_{1}(\lambda) \| h_{1}(\mu)$. One of the following situations may occur:

- $\beta_{0} \leqslant_{\mathbb{L}} \lambda$ and $\beta_{0} \leqslant_{\mathbb{L}} \mu$. In such a case, we get $h_{1}(\lambda)=h_{0}(\lambda) \cup\left\{x_{n+1}\right\}$ and $h_{1}(\mu)=$ $h_{0}(\mu) \cup\left\{x_{n+1}\right\}$, so $h_{1}(\lambda)$ and $h_{1}(\mu)$ are non-comparable since $h_{0}(\lambda)$ and $h_{0}(\mu)$ are also non-comparable.

- $\beta_{0} \mathbb{E}_{\mathbb{L}} \lambda$ and $\beta_{0} \mathbb{E}_{\mathbb{L}} \mu$. In such a case, similarly to the previous situation, the noncomparability of $h_{0}(\lambda)$ and $h_{0}(\mu)$ implies that $h_{1}(\lambda)$ and $h_{1}(\mu)$ are non-comparable since $h_{1}(\lambda)=h_{0}(\lambda)$ and $h_{1}(\mu)=h_{0}(\mu)$.

- $\beta_{0} \leqslant_{\mathbb{L}} \lambda$ and $\beta_{0} \mathbb{E}_{\mathbb{L}} \mu$ (or $\beta_{0} \mathbb{L}_{\mathbb{L}} \lambda$ and $\beta_{0} \leqslant_{\mathbb{L}} \mu$ ). Without loss of generality, assume that $\beta_{0} \leqslant \mathbb{L} \lambda$ and $\beta_{0} \mathbb{L}_{\mathbb{L}} \mu$. Then $h_{1}(\lambda)=h_{0}(\lambda) \cup\left\{x_{n+1}\right\}$ and $h_{1}(\mu)=h_{0}(\mu)$. Also in this case, since $h_{0}(\lambda)$ and $h_{0}(\mu)$ are non-comparable, also $h_{1}(\lambda)$ and $h_{1}(\mu)$ are. This proves that $h_{1}$ is $\{\lambda, \mu\}$-preserving.

Now, we prove that $h_{1}$ is a $\psi$-complementary map. As $\alpha<_{\mathbb{L}} \beta_{0}$, we must have that $\beta_{0} \neq \hat{0}_{\mathbb{L}}$ and that $\beta_{0} \mathbb{E}_{\mathbb{L}} \gamma$ or $\beta_{0} \mathbb{K}_{\mathbb{L}} \psi(\gamma)$. To prove that $h_{1}$ is a $\psi$-complementary map, we shall demonstrate that:

(i) $h_{1}(\gamma)=\Omega_{n+1} \backslash h_{1}(\psi(\gamma))$ if either $\beta_{0} \leqslant \mathbb{L} \gamma$ or $\beta_{0} \leqslant \mathbb{L} \psi(\gamma)$;

(ii) $h_{1}(\gamma) \varsubsetneqq \Omega_{n+1} \backslash h_{1}(\psi(\gamma))$ if $\beta_{0} \mathbb{E}_{\mathbb{L}} \gamma$ and $\beta_{0} \mathbb{K}_{\mathbb{L}} \psi(\gamma)$.

To show $(i)$, assume firstly that $\beta_{0} \leqslant \mathbb{L} \gamma$. Then $\beta_{0} \mathbb{E}_{\mathbb{L}} \psi(\gamma)$ and

$$
h_{1}(\gamma)=h_{0}(\gamma) \cup\left\{x_{n+1}\right\}=\left(\Omega_{n} \backslash h_{0}(\psi(\gamma))\right) \cup\left\{x_{n+1}\right\}=\Omega_{n+1} \backslash h_{1}(\psi(\gamma)) .
$$

Similarly, assume that $\beta_{0} \leqslant_{\mathbb{L}} \psi(\gamma)$. Then $\beta_{0} \mathbb{E}_{\mathbb{L}} \gamma$ and

$$
h_{1}(\gamma)=h_{0}(\gamma)=\Omega_{n} \backslash h_{0}(\psi(\gamma))=\Omega_{n+1} \backslash h_{1}(\psi(\gamma)) .
$$


In case $(i i)$, if we take $\gamma$ such that $\beta_{0} \mathbb{L}_{\mathbb{L}} \gamma$ and $\beta_{0} \mathbb{E}_{\mathbb{L}} \psi(\gamma)$, then

$$
h_{1}(\gamma)=h_{0}(\gamma)=\Omega_{n} \backslash h_{0}(\psi(\gamma)) \varsubsetneqq \Omega_{n+1} \backslash h_{1}(\psi(\gamma)) .
$$

Therefore, set

$$
\Gamma_{1}:=\left\{\gamma \in L: \beta_{0} \mathbb{E}_{\mathbb{L}} \gamma, \beta_{0} \mathbb{K}_{\mathbb{L}} \psi(\gamma)\right\} .
$$

If $\Gamma_{1}=\emptyset$, we set $g:=h_{1}, \Omega:=\Omega_{n+1}$ and $\mathcal{G}:=\operatorname{Im}(g)$. Then, $\mathcal{G}$ is a complement-closed set system on $\Omega$. Moreover, $g(\alpha) \varsubsetneqq g(\beta)$, i.e. $g$ is $\{\alpha, \beta\}$-preserving. Hence the set $\Omega$, the set system $\mathcal{G}$ and the map $g$ satisfy the conditions of the statement and the proof concludes here.

Otherwise, if $\Gamma_{1} \neq \emptyset$, we fix an arbitrary element $\beta_{1} \in \Gamma_{1}$ and we consider the map $\gamma_{2}: L \rightarrow \mathcal{P}\left(\Omega_{n+1}\right)$ given by

$$
h_{2}(\gamma):= \begin{cases}h_{1}(\gamma) \cup\left\{x_{n+1}\right\} & \text { if } \beta_{1} \leqslant \mathbb{L} \gamma \\ h_{1}(\gamma) & \text { otherwise }\end{cases}
$$

for any $\gamma \in L$.

Then, reasoning as for the map $h_{1}$, it may be easily proved that $h_{2}$ is order-preserving and that if $h_{1}$ is $\{\lambda, \mu\}$-preserving, then also $h_{2}$ is $\{\lambda, \mu\}$-preserving. Moreover $h_{2}(\alpha) \varsubsetneqq h_{2}(\beta)$. Set

$$
\Gamma_{2}:=\left\{\gamma \in \Gamma_{1}: \beta_{1} \mathbb{K}_{\mathbb{L}} \gamma, \beta_{1} \mathbb{K}_{\mathbb{L}} \psi(\gamma)\right\} .
$$

As before, note that if $\gamma \notin \Gamma_{2}$, then

$$
h_{2}(\gamma)=\Omega_{n+1} \backslash h_{2}(\psi(\gamma))
$$

while if $\gamma \in \Gamma_{2}$, then we get

$$
h_{2}(\gamma) \varsubsetneqq \Omega_{n+1} \backslash h_{2}(\psi(\gamma)) .
$$

Furthermore, note that $\Gamma_{2} \varsubsetneqq \Gamma_{1}$ because $\beta_{1} \in \Gamma_{1} \backslash \Gamma_{2}$. At this point, we can reiterate the previous construction in order to obtain a sequence of functions $h_{i}$ and of subsets $\Gamma_{i}$ of $L$ such that each $h_{i}$ is order-preserving and $\{\alpha, \beta\}$-preserving and $\Gamma_{i} \varsubsetneqq \Gamma_{i-1}$ for any index $i \geqslant 2$.

As $L$ is finite, there must be some index $k$, and hence a subset $\Gamma_{k}$ which must be empty. In other terms, for each $\gamma \in L$, it must be

$$
h_{k}(\gamma)=\Omega_{n+1} \backslash h_{k}(\psi(\gamma)) .
$$

At this point, we get the thesis taking $g:=h_{k}, \Omega:=\Omega_{n+1}$ and $\mathcal{G}:=\operatorname{Im}(g) \subseteq \mathcal{P}(\Omega)$.

$(C 2)$ : The proof is the same as that given in $(C 1)$, after reversing the role of $\alpha$ and $\beta$. $(C 3)$ : Also in this case, we take $\Omega_{n+1}, h_{0}, \beta_{0}$ and $h_{1}$ as in the case $(C 1)$. Then, as $\alpha \| \beta$, we easily prove as in $(C 1)$ that $h_{1}$ is a $\psi$-complementary map. Therefore, also in the present case we can define the sequence of subsets $\Gamma_{i}$ and the sequence of functions $h_{i}$ as in $(C 1)$. Again, let $k$ be the minimum integer for which $\Gamma_{k}=\emptyset$, so that

$$
h_{k}(\gamma)=\Omega_{n+1} \backslash h_{k}(\psi(\gamma)) .
$$


Then, in this case we obtain $h_{k}(\alpha) \varsubsetneqq h_{k}(\beta)$. Thus, $h_{k}$ is not $\{\alpha, \beta\}$-preserving. Consider an element $x_{n+2} \notin \Omega_{n+1}$ and set $\Omega_{n+2}:=\Omega_{n+1} \cup\left\{x_{n+2}\right\}$. We set $\bar{h}_{0}:=h_{k}, \alpha_{0}:=\alpha$ and consider the map $\bar{h}_{1}: L \rightarrow \mathcal{P}\left(\Omega_{n+2}\right)$ given by

$$
\bar{h}_{1}(\gamma):= \begin{cases}\bar{h}_{0}(\gamma) \cup\left\{x_{n+2}\right\} & \text { if } \alpha_{0} \leqslant \mathbb{L} \gamma \\ \bar{h}_{0}(\gamma) & \text { otherwise }\end{cases}
$$

for any $\gamma \in L$.

Note that the map $\bar{h}_{1}$ is $\{\alpha, \beta\}$-preserving. Furthermore, it results that $\alpha_{0} \neq \hat{0}_{\mathbb{L}}$ since $\alpha_{0} \| \beta$. Moreover, since $\psi(\delta) \wedge \delta=\hat{0}_{\mathbb{L}}$ for any $\delta \in L$, we get $\alpha \mathbb{L}_{\mathbb{L}} \gamma$ or $\alpha \mathbb{L}_{\mathbb{L}} \psi(\gamma)$ for any $\gamma \in L$.

At this point, we claim that if $\alpha_{0} \leqslant \mathbb{L} \gamma$ or $\alpha_{0} \leqslant \mathbb{L} \psi(\gamma)$, then

$$
\bar{h}_{1}(\gamma)=\Omega_{n+2} \backslash \bar{h}_{1}(\psi(\gamma)) .
$$

To this regard, let $\alpha_{0} \leqslant_{\mathbb{L}} \gamma$. Then $\alpha_{0} \mathbb{K}_{\mathbb{L}} \psi(\gamma)$ and

$$
\begin{gathered}
\bar{h}_{1}(\gamma)=\bar{h}_{0}(\gamma) \cup\left\{x_{n+2}\right\}=h_{k}(\gamma) \cup\left\{x_{n+2}\right\}=\left(\Omega_{n+1} \backslash h_{k}(\psi(\gamma))\right) \cup\left\{x_{n+2}\right\}= \\
=\Omega_{n+2} \backslash h_{k}(\psi(\gamma))=\Omega_{n+2} \backslash \bar{h}_{0}(\psi(\gamma))=\Omega_{n+2} \backslash \bar{h}_{1}(\psi(\gamma)),
\end{gathered}
$$

as $h_{k}(\gamma)=\Omega_{n+1} \backslash h_{k}(\psi(\gamma))$ for each $\gamma \in L$. Similarly, if $\left.\alpha_{0} \leqslant_{\mathbb{L}} \psi(\gamma)\right)$ and $\alpha_{0} \mathbb{E}_{\mathbb{L}} \gamma$, then

$$
\begin{aligned}
\bar{h}_{1}(\gamma) & =\bar{h}_{0}(\gamma)=h_{k}(\gamma)=\Omega_{n+1} \backslash h_{k}(\psi(\gamma))= \\
& =\Omega_{n+1} \backslash \bar{h}_{0}(\psi(\gamma))=\Omega_{n+2} \backslash \bar{h}_{1}(\psi(\gamma)) .
\end{aligned}
$$

Finally, assume that $\alpha_{0} \mathbb{L}_{\mathbb{L}} \gamma$ and $\alpha_{0} \mathbb{E}_{\mathbb{L}} \psi(\gamma)$. In such a case, we get

$$
\begin{aligned}
& \bar{h}_{1}(\gamma)= \bar{h}_{0}(\gamma)=h_{k}(\gamma)=\Omega_{n+1} \backslash h_{k}(\psi(\gamma))= \\
&=\Omega_{n+1} \backslash \bar{h}_{0}(\psi(\gamma)) \varsubsetneqq \Omega_{n+2} \backslash \bar{h}_{1}(\psi(\gamma))
\end{aligned}
$$

At this point, set

$$
\bar{\Gamma}_{1}:=\left\{\gamma \in L: \alpha_{0} \mathbb{Z}_{\mathbb{L}} \gamma, \alpha_{0} \mathbb{K}_{\mathbb{L}} \psi(\gamma)\right\} .
$$

If $\bar{\Gamma}_{1}=\emptyset$, just set $g:=\bar{h}_{1}, \Omega:=\Omega_{n+2}$ and $\mathcal{G}:=\operatorname{Im}(g)$. Clearly, $\mathcal{G}$ is complement-closed on $\Omega$ and the theorem is proved.

Otherwise, let $\alpha_{1} \in \bar{\Gamma}_{1}$ and set

$$
\bar{h}_{2}(\gamma):= \begin{cases}\bar{h}_{1}(\gamma) \cup\left\{x_{n+2}\right\} & \text { if } \alpha_{1} \leqslant \mathbb{L} \gamma \\ \bar{h}_{1}(\gamma) & \text { otherwise }\end{cases}
$$

for any $\gamma \in L$. The map $\bar{h}_{2}$ is clearly order-preserving as $\bar{h}_{1}$ is and it is also $\{\alpha, \beta\}$ preserving. Set

$$
\bar{\Gamma}_{2}:=\left\{\gamma \in \bar{\Gamma}: \alpha_{1} \mathbb{K}_{\mathbb{L}} \gamma, \alpha_{1} \mathbb{K}_{\mathbb{L}} \psi(\gamma)\right\} .
$$

Note that if $\gamma \notin \bar{\Gamma}_{2}$, then it may be easily shown that

$$
\bar{h}_{2}(\gamma)=\Omega_{n+2} \backslash \bar{h}(\psi(\gamma))
$$


while if $\gamma \in \bar{\Gamma}_{2}$, we have that

$$
\bar{h}_{2}(\gamma) \varsubsetneqq \Omega_{n+2} \backslash \bar{h}(\psi(\gamma)) .
$$

Furthermore, notice that $\bar{\Gamma}_{2} \varsubsetneqq \bar{\Gamma}_{1}$ since $\alpha_{1} \in \bar{\Gamma}_{1} \backslash \bar{\Gamma}_{2}$. Iterating the above construction, we obtain a sequence of maps $\bar{h}_{i}$ and of subsets $\bar{\Gamma}_{i}$ of $L$ such that each $\bar{h}_{i}$ is an orderpreserving map and also an $\{\alpha, \beta\}$-preserving map and $\bar{\Gamma}_{i} \varsubsetneqq \bar{\Gamma}_{i-1}$ for each index $i \geqslant 2$.

As $L$ is finite, we will find an index $s$ for which $\bar{\Gamma}_{s}=\emptyset$. We get the thesis taking $g:=\bar{h}_{s}$, $\Omega:=\Omega_{n+2}$ and $\mathcal{G}=\operatorname{Im}(g) \subseteq \mathcal{P}(\Omega)$.

$(C 4)$ : Just set $\bar{h}_{0}:=f$ and repeat the construction given in $(C 3)$.

$(C 5)$ : Taking both the cases $(C 3)$ and $(C 4)$, we showed that the thesis follows when $\alpha \| \beta$ and $f(\alpha) \subseteq f(\beta)$. Therefore, if $\alpha \| \beta$ and $f(\beta) \varsubsetneqq f(\alpha)$, we may proceed as in $(C 4)$ reversing the role $\alpha$ of $\beta$.

In the next result, we prove that for any finite complemented involutory lattice there exists a pairing on a certain finite set $\Omega_{\mathbb{L}, \psi}$ whose normally extensible family is isomorphic to $\mathbb{L}$ itself.

Theorem 22. Let $(\mathbb{L}, \psi)$ be a finite complemented involutory lattice. Then there exist a finite set $\Omega_{\mathbb{L}, \psi}$ and a regular pairing $\mathcal{W}$ on $\Omega_{\mathbb{L}, \psi}$ such that $\mathbb{E}(\mathcal{W})$ is order-isomorphic to $\mathbb{L}$.

Proof. The idea of the proof is the following: first of all, we want to show the existence of a finite set $\Omega_{\mathbb{L}, \psi}$ and of a complement-closed family $\mathcal{F}$ on $\Omega_{\mathbb{L}, \psi}$ such that $\mathbb{L}$ is orderisomorphic to $(\mathcal{F}, \subseteq)$; secondly, we want to show the existence of some regular pairing $\mathcal{W}$ on $\Omega_{\mathbb{L}, \psi}$ such that $\mathcal{F}=\mathcal{E}(\mathcal{W})$. To this regard, we will divide our proof in two steps:

$(i)$ : We shall construct a finite set $\Omega_{\mathbb{L}, \psi}$ and a complement-closed family $\mathcal{F}$ on $\Omega_{\mathbb{L}, \psi}$ such that an isomorphism $f: \mathbb{L} \rightarrow(\mathcal{F}, \subseteq)$ exists. Firstly, set $\Lambda_{0}:=\emptyset, \mathcal{F}_{0}:=\{\emptyset\}$ and $f_{0}: L \rightarrow \mathcal{F}_{0}$ is the constant map on $L$ such that $f(\gamma)=\emptyset$ for each $\gamma \in L$. Note that $\mathcal{F}_{0}$ is a complementclosed family on $\Lambda_{0}$ and that $f_{0}$ is trivially an order-preserving map from $\mathbb{L}$ onto $\left(\mathcal{F}_{0}, \subseteq\right)$. If $|L|=1$, the thesis has been proved just taking $\Omega_{\mathbb{L}, \psi}:=\Lambda_{0}$ and $\mathcal{G}:=\{\emptyset\}$ and $g:=f_{0}$ as our isomorphism. Therefore, in what follows, we may assume that $l:=|L| \geqslant 2$. Let us consider the following subset of pairs of $L$ :

$$
\mathcal{H}_{0}:=\left\{(\alpha, \beta) \in L^{2}: \alpha \neq \beta, f_{0} \text { is not }\{\alpha, \beta\} \text {-preserving }\right\} .
$$

Clearly, the number of elements of $\mathcal{H}_{0}$ is finite and equals $\left(\begin{array}{l}l \\ 2\end{array}\right)$. Fix now $\left(\alpha_{0}, \beta_{0}\right) \in \mathcal{H}_{0}$. In view of Theorem 21 there exist a finite set $\Omega$, a complement-closed family $\mathcal{G}$ on $\Omega$ and an order-preserving, $\psi$-complementary and $\left\{\alpha_{0}, \beta_{0}\right\}$-preserving map $g: \mathbb{L} \rightarrow(\mathcal{G}, \subseteq)$. Set $\Lambda_{1}:=\Omega, \mathcal{F}_{1}:=\mathcal{G}$ and $f_{1}:=g$ and define the following subset of pairs of $L$ :

$$
\mathcal{H}_{1}:=\left\{(\alpha, \beta) \in \mathcal{H}_{0}: \alpha \neq \beta, f_{1} \text { is not }\{\alpha, \beta\} \text {-preserving }\right\} .
$$

Notice that $\mathcal{H}_{1} \varsubsetneqq \mathcal{H}_{0}$ as $\left(\alpha_{0}, \beta_{0}\right) \in \mathcal{H}_{0} \backslash \mathcal{H}_{1}$. At this point, fix $\left(\alpha_{1}, \beta_{1}\right) \in \mathcal{H}_{1}$. In view of Theorem 21 there exist a finite set $\Omega$, a complement-closed family $\mathcal{G}$ on $\Omega$ and an orderpreserving, $\psi$-complementary and $\left\{\alpha_{1}, \beta_{1}\right\}$-preserving map $g: \mathbb{L} \rightarrow(\mathcal{G}, \subseteq)$.

Set $\Lambda_{2}:=\Omega, \mathcal{F}_{2}:=\mathcal{G}$ and $f_{2}:=g$ and define the following subset of pairs of $L$ :

$$
\mathcal{H}_{2}:=\left\{(\alpha, \beta) \in \mathcal{H}_{1}: \alpha \neq \beta, f_{2} \text { is not }\{\alpha, \beta\} \text {-preserving }\right\} .
$$


Notice that $\mathcal{H}_{2} \varsubsetneqq \mathcal{H}_{1}$ as $\left(\alpha_{1}, \beta_{2}\right) \in \mathcal{H}_{2} \backslash \mathcal{H}_{1}$. Furthermore, let us notice that if $(\lambda, \mu) \in$ $L^{2} \backslash \mathcal{H}_{2}$ and $\lambda \neq \mu$, then the map $f_{1}$ is $\{\lambda, \mu\}$-preserving and Theorem 21 ensures that $f_{2}$ is also $\{\lambda, \mu\}$-preserving.

Therefore, we may iterate the above construction to obtain a strictly decreasing sequence

$$
\mathcal{H}_{0} \supsetneqq \mathcal{H}_{1} \supsetneqq \cdots \supsetneqq \mathcal{H}_{i} \supsetneqq \cdots
$$

of subsets of $L^{2}$ which, in view of the finiteness of $L^{2}$, must terminate with $\mathcal{H}_{k}=\emptyset$ for some integer $k$. Take $\Omega_{\mathbb{L}, \psi}:=\Lambda_{k}, \mathcal{F}:=\mathcal{F}_{k}$ and $f:=f_{k}$. Clearly, in view of Theorem 21, it follows that $f$ is order-preserving and that $\mathcal{F}=\operatorname{Im}(f)$. Moreover, $f$ is both injective and surjective, since it is $\{\alpha, \beta\}$-preserving for each $\alpha, \beta \in L$.

The fact that $f$ is an $\{\alpha, \beta\}$-preserving map also ensures that $f^{-1}$ is also order-preserving, i.e. $f$ is an isomorphism between the posets $\mathbb{L}$ and $(\mathcal{F}, \subseteq)$. Thus, the isomorphism $f: \mathbb{L} \rightarrow(\mathcal{F}, \subseteq)$ induces on $(\mathcal{F}, \subseteq)$ a natural lattice structure.

(ii): At this point, we want to show the existence of a regular pairing $\mathcal{W}$ on $\Omega_{\mathbb{L}, \psi}$ such that $\mathcal{F}=\mathcal{E}(\mathcal{W})$.

Let us consider the set operator $\operatorname{Int}_{\mathcal{F}}: \mathcal{P}\left(\Omega_{\mathbb{L}, \psi}\right) \rightarrow \mathcal{P}\left(\Omega_{\mathbb{L}, \psi}\right)$. In view of Proposition 2 , it results that $I n t_{\mathcal{F}}$ is a closure operator such that $\operatorname{Int}_{\mathcal{F}}(\emptyset)=\emptyset$ and, hence, Proposition 12 ensures the existence of a regular pairing $\mathcal{W}$ on $\Omega_{\mathbb{L}, \psi}$ such that $\mathcal{M}(\mathcal{W})=F i x\left(\right.$ Int $\left._{\mathcal{F}}\right)$. Furthermore, it results that $\operatorname{Int}_{\mathcal{F}}=M_{\mathcal{W}}$ in view of Theorem 1 . We must prove that $\mathcal{F}=\mathcal{E}(\mathcal{W})$. We shall firstly show the inclusion $\mathcal{F} \subseteq \mathcal{E}(\mathcal{W})$. To this regard fix $Z \in \mathcal{F}$. Proposition 12 ensures that $K_{\mathcal{W}}(Z)=Z$, so we get

$$
Z=N_{\mathcal{W}}(Z)
$$

i.e. $Z \in \mathcal{E}(\mathcal{W})$.

On the other hand, we shall demonstrate that $\mathcal{E}(\mathcal{W}) \subseteq \mathcal{F}$. So, let $Z \in \mathcal{E}(\mathcal{W})$. Clearly, $Z \in \mathcal{M}(\mathcal{W})$. Now, since $\mathcal{F}$ is a complete lattice, it follows that

$$
\left.Z^{*}:=\bigwedge Y \in \mathcal{F}: Z \subseteq Y\right\} \in \mathcal{F} .
$$

Furthermore, we have that

$$
Z^{*} \subseteq \bigcap\{Y \in \mathcal{F}: Z \subseteq Y\}=\operatorname{Int}_{\mathcal{F}}(Z)=M_{\mathcal{W}}(Z)=Z .
$$

We claim that $Z=Z^{*}$. In view of Proposition 12 , we easily note that $\left(Z^{*}\right)^{c} \in \mathcal{M}(\mathcal{W})$. Furthermore, let $A \subseteq Z$ be such that $A^{c} \in \mathcal{M}(\mathcal{W})$. We claim that

$$
A=\bigcup\{Y \in \mathcal{F}: Y \subseteq A\} .
$$

To this regard, just notice that $A^{c}=M_{\mathcal{W}}\left(A^{c}\right)=\operatorname{Int}_{\mathcal{F}}\left(A^{c}\right)=\bigcap\left\{Y \in \mathcal{F}: A^{c} \subseteq Y\right\}$, whence, passing to the complements, we infer the validity of (35), as wanted.

Clearly, in view of (34), we easily deduce that $Y \subseteq Z^{*}$ for each subset $Y \in \mathcal{F}$ which is also contained in $A$. In particular, this implies that $A \subseteq Z^{*}$. In other terms, we proved that $Z^{*}$ is the greatest subset of $Z$ whose complement is a maximum partitioner. So, $K_{\mathcal{W}}(Z)=Z^{*}$ in view of $(i)$ of Proposition 11. As $K_{\mathcal{W}}(Z)=Z^{*} \in \mathcal{F}$, we get the following identities:

$$
Z=N_{\mathcal{W}}(Z)=M_{\mathcal{W}}\left(Z^{*}\right)=Z^{*}
$$

whence we deduce that $Z \in \mathcal{F}$. So, $\mathcal{F}=\mathcal{E}(\mathcal{W})$ and the proof concludes here. 


\section{Conclusions}

In this paper we continued the investigations of some links between granular computing $(\mathrm{GrC})$, closure systems and algebraic order theory. To this regard, the notion of pairing becomes a basic tool of our analysis.

In the perspective of the present work, a pairing $\mathcal{W}=\langle F, U, \Omega, \Lambda\rangle$ has been considered as a purely mathematical interpretation of the classical notions of information system [28] and relational data table [34]. We used the set partitions induced by the map $F$ : $U \times \Omega \longrightarrow \Lambda$ in order to define a specific closure system $\mathcal{M}(\mathcal{W})$ (whose members are called maximum partitioners), and a related closure operator $M_{\mathcal{W}}$ on the set $\Omega$. By means of such an operator, one can describe the classical functional dependency between attribute subsets of $\Omega$, when this set is interpreted as an attribute set of some information system. Now, since several constructions used in database theory have a natural lattice structure (see [34] for details), it is natural to investigate the links between lattices and the above closure system induced by pairings. These links may be obtained by means of a binary relation $\geqslant$ between subsets of $\Omega$, which we called union additive relation. Any $\geqslant$ can be represented as a preorder $\geqslant_{\mathcal{W}}$ induced by some pairing $\mathcal{W}$ on the same ground set $\Omega$. This implies that pairings are concrete models describing the union additive relations. Then, in this paper we proved three main representation results.

The first of such results is Theorem 5. In this theorem we showed that any closure system $\mathcal{S}$ on the set $\Omega$ can be described as the family of all maximum partitioners $\mathcal{M}(\mathcal{W})$ of some pairing $\mathcal{W}$ on $\Omega$. The proof given in Theorem 5 is constructive, and by means of it we can explicitly provide a pairing $\mathcal{W}$ on $\Omega$ such that $\mathcal{M}(\mathcal{W})=\mathcal{S}$.

The second representation result is Theorem 17, which concerns finite lattices and that is proved by means of Theorem 5. In such a theorem we showed that any finite lattice $\mathbb{L}$ coincides with the maximum partitioner lattice of some pairing on the set $\Omega_{\mathbb{L}}$ of all join-irreducible elements of $\mathbb{L}$. As a direct consequence, we see that the partial order of any finite lattice can be refined by means of a numerical map $\gamma_{\mathcal{W}}$ associated with the closure system $\mathcal{N}(\mathcal{W})$. In fact, by means of the map $\gamma_{\mathcal{W}}$ we can also establish a partial dependency level between non-comparable nodes of the lattice.

Eventually, in Section 5 we studied a specific class of finite lattices, which we called complemented involutory lattices, i.e. lattices $\mathbb{L}=\left(L, \leqslant_{\mathbb{L}}\right)$ endowed with an orderreversing involutory map $\psi: L \rightarrow L$ exchanging $\hat{0}_{\mathbb{L}}$ and $\hat{1}_{\mathbb{L}}$ and such that $\psi(\alpha) \wedge \alpha=\hat{0}_{\mathbb{L}}$ and $\psi(\alpha) \vee \alpha=\hat{1}_{\mathbb{L}}$. Also for such types of lattices we provided a representation result in terms of pairings, which is Theorem 22. In this theorem we found a finite set $\Omega_{\mathbb{L}, \psi}$ and a pairing $\mathcal{W}$ on $\Omega_{\mathbb{L}, \psi}$ such that the poset induced by the set system $\mathcal{E}(\mathcal{W})=F i x\left(M_{\mathcal{W}} \circ K_{\mathcal{W}}\right)$ (where $K_{\mathcal{W}}$ is the set operator defined by $K_{\mathcal{W}}(A):=\Omega \backslash M_{\mathcal{W}}\left(A^{c}\right)$ ) is order-isomorphic to $\mathbb{L}$.

As a future research perspective, we can start from the observation that there is not a uniquely determined way to associate a pairing with a given closure system $\mathcal{S}$. Therefore one could study the relations between all pairings $\mathcal{W}$ on $\Omega$ for which $\mathcal{M}(\mathcal{W})=\mathcal{S}$, in terms of their corresponding maps $\gamma_{\mathfrak{P}}$. Closely related to the previous question is the problem of determing a sort of canonical pairing associated with the lattice $\mathbb{L}$. 
By the way, we think that the aforementioned theorems represent a useful starting point to frame in an unifying perspective the abstract analysis of lattices with motivations related to the functional dependency in database theory.

\section{Acknowledgements}

We are extremely grateful to the anonymous referee who helped us to improve the quality of our paper with his thorough suggestions.

\section{References}

[1] J. A. Aledo, L. G. Diaz, S. Martinez, J. C. Valverde, Predecessors and Garden-ofEden configurations in parallel dynamical systems on maxterm and minterm Boolean functions, Journal of Computational and Applied Mathematics (2019) 348, 26-33.

[2] J. A. Aledo, L. G. Diaz, S. Martinez, J.C. Valverde, Solution to the predecessors and Gardens-of-Eden problems for synchronous systems over directed graphs, Applied Mathematics and Computation, 347 (2019), 22-28.

[3] V. B. Alekseev, The number of families of subsets that are closed with respect to intersections, Diskretnaya Matematika 1(2), 129-136 (1989).

[4] K. J. Al-Agha, R. J. Greechie, The involutory dimension of involution posets, Order, 18 (2001), no.4, 323-337 (2002).

[5] C. Berge, Hypergraphs: Combinatorics of Finite Sets, Elsevier, Amsterdam, 1984.

[6] G. Birkhoff, Lattice Theory, American Mathematical Society, Providence, Rhode Island, Third Edition, 1967.

[7] C. Bisi, On Commuting Polynomial Automorphisms of $\mathbb{C}^{k}, k \geqslant 3$, Mathematische Zeitschrift, 258(4) (2008), 875-891.

[8] C. Bisi, On Closed Invariant Sets in Local Dynamics, Journal of Mathematical Analysis and Applications, 350(1) (2009), 327-332.

[9] P. Bonacini, M. Gionfriddo, L. Marino, Nesting House-designs, Discrete Mathematics 339 (4) (2016), 1291-1299.

[10] G. Cattaneo, G. Chiaselotti, P. A. Oliverio, F. Stumbo, A New Discrete Dynamical System of Signed Integer Partitions, European Journal of Combinatorics 55 (2016), 119-143.

[11] I. Chajda, H. Länger, Symmetric Differences on Posets with an Antitone Involution, Order, 29, no. 1, 215-225 (2012).

[12] G. Chiaselotti, D. Ciucci, T. Gentile, F. Infusino, The Granular Partition Lattice of an Information Table, Information Sciences 373 (2016), 57-78.

[13] G. Chiaselotti, T. Gentile, F. Infusino, P. A. Oliverio, The Adjacency Matrix of a Graph as a Data Table. A Geometric Perspective, Annali di Matematica Pura e Applicata, Vol. 196, No. 3, (2017), 1073-1112. 
[14] G. Chiaselotti, T. Gentile, F. Infusino, Simplicial Complexes and Closure Systems induced by Indistinguishability Relations., C. R. Acad. Sci. Paris, Ser. I, 355 (2017), 991-1021.

[15] G. Chiaselotti, T. Gentile, F. Infusino, Dependency Structures for Decision Tables, International Journal of Approximate Reasoning, 88 (2017), 333-370.

[16] G. Chiaselotti, T. Gentile, F. Infusino, P. A. Oliverio, Dependency and Accuracy Measures for Directed Graphs, Applied Mathematics and Computation, 320 (2018), 781-794.

[17] G. Chiaselotti, F. Infusino, Notions from Rough Set Theory in a Generalized Dependency Relation Context, International Journal of Approximate Reasoning 98 (2018), 25-61.

[18] G. Chiaselotti, T. Gentile, F. Infusino, Local Dissymmetry on Graphs and Related Algebraic Structures, International Journal of Algebra and Computation, Vol. 29, No. 8, 2019, 1499-1526.

[19] B. A. Davey, H. A. Priestley, Introduction to lattices and order, Second edition. Cambridge University Press, New York, (2002).

[20] M. Droste, G. -Q. Zhang, Bifinite Chu Spaces, Logical Methods in Computer Science, Vol. 6 (1:3) 2010, pp. 1-20.

[21] K. Engel, T. D. Thu, Boundary optimization for rough sets. Discrete Mathematics, 341 (2018), 2465-2477.

[22] Z. Frolik, M. Katetov, V. Ptak, General Topology and its Relations to Modern Analysis and Algebra II, Proceedings of the Second Prague Topological Symposium, Academic Press, New York, 1966.

[23] M. Gionfriddo, E. Guardo, L. Milazzo, Extending bicolorings for Steiner triple systems, Applicable Analysis and Discrete Mathematics (2013), 225-234.

[24] M. F. Janowitz, On the Antitone Mappings of a Poset, Proceedings of the American Mathematical Society (1964), no.15, no. 4, 529-533.

[25] X. Li, S. Liu, Matroidal approaches to rough sets via closure operators, International Journal of Approximate Reasoning, 53 (2012) 513-527.

[26] X. Li, H. Yi, S. Liu, Rough Sets and Matroids from a Lattice-Theoretic Viewpoint, Information Sciences, 342 (2016) 37-52.

[27] E. H. Moore, Introduction to a form of general analysis, vol. 2, Yale University Press (1910).

[28] Z. Pawlak, Rough sets. Theoretical Aspects of Reasoning about Data, Kluwer Academic Publisher, 1991.

[29] W. Pedrycz, Granular Computing: An Emerging Paradigm, Springer-Verlag, Berlin (2001).

[30] W. Pedrycz, Granular Computing for Data Analytics: A Manifesto of Human-Centric Computing, EEE/CAA Journal of Automatica Sinica, Vol.5, No.6, November 2018, 1025-1034. 
[31] P. Ribemboimb, Algebraic Structures on Graphs, Algebra Universalis, 16 (1983) 105-123.

[32] G. T. Rüttimann, Closure operators and projections on involution posets, Journal of Australian Mathematical Society, 18, Issue 04, 453-457 (1974).

[33] S. M. Sanahuja, New rough approximations for $n$-cycles and n-paths, Applied Mathematics and Computation, (2016) 276, 96-108.

[34] D. A. Simovici, C. Djeraba, Mathematical Tools for Data Mining, Springer-Verlag, London 2014.

[35] G. Hahn and G. Sabidussi (Editors); Graph Symmetry. Algebraic Methods and Applications; NATO ASI Series, Vol.497, 1997, Springer.

[36] J. Tanga, K. Shea, F. Min, W. Zhu, A matroidal approach to rough set theory, Theoretical Computer Science, Volume 471, 3 February 2013, Pages 1-11.

[37] J. Wang, W. Zhu, Applications of Bipartite Graphs and their Adjacency Matrices to Covering-based Rough Sets, Fundamenta Informaticae, 156 (2017), 237-254.

[38] Y. Y. Yao, N.Zhong, Granular Computing using Information Tables, in Data Mining, Rough Sets and Granular Computing, Physica-Verlag, 2002, pp. 102-124.

[39] Y. Yao, A Partition Model of Granular Computing, in: Transactions on Rough Sets I, Lecture Notes in Computer Science, vol. 3100, Springer-Verlag, 2004, 232-253. 